\title{
Pacific
}

Journal of

Mathematics

\section{AN EXTENSION OF THE QUINTUPLE PRODUCT IDENTITY AND ITS APPLICATIONS}

\section{ZHI-GUO LIU}




\title{
AN EXTENSION OF THE QUINTUPLE PRODUCT IDENTITY AND ITS APPLICATIONS
}

\author{
ZHI-GUO LIU
}

\begin{abstract}
Using the theory of elliptic theta functions, we establish a theta function identity that may be regarded as an extension of the quintuple identity, with many other results, both classical and new, included as special cases. It allows us to give a new derivation of the Ramanujan-Watson modular equation of the seventh order. We give new proofs of some Eisenstein series identities of Ramanujan related to modular equations of degree 7.
\end{abstract}

\section{Introduction}

Throughout we put $q=e^{2 \pi i \tau}$, where $\operatorname{Im} \tau>0$.

Definition 1.1. The Jacobi theta functions $\theta_{k}$ for $k=1,2,3,4$ are defined as

$$
\begin{aligned}
& \theta_{1}(z \mid \tau)=2 \sum_{n=0}^{\infty}(-1)^{n} q^{(2 n+1)^{2} / 8} \sin (2 n+1) z \\
& \theta_{2}(z \mid \tau)=2 \sum_{n=0}^{\infty} q^{(2 n+1)^{2} / 8} \cos (2 n+1) z \\
& \theta_{3}(z \mid \tau)=1+2 \sum_{n=1}^{\infty} q^{n^{2} / 2} e^{2 n i z} \\
& \theta_{4}(z \mid \tau)=1+2 \sum_{n=1}^{\infty}(-1)^{n} q^{n^{2} / 2} e^{2 n i z}
\end{aligned}
$$

These series converge for all complex $z$ whenever $\operatorname{Im} \tau>0$, and they converge absolutely and uniformly on compact subsets and so are entire functions of $z$.

MSC2000: 11F11, 11F20, 11F27, 33E05.

Keywords: elliptic function, theta function, modular equation, Eisenstein series, Jacobi's quartic identity, sum of squares, Ramanujan's identities, quintuple product identity.

Supported by the National Science Foundation of China, the PCSIRT, the Shanghai Natural Science Foundation, grant number 10ZR1409100, and the Shanghai Leading Academic Discipline Project, project number B407. 
The well-known Jacobi triple product identity [Andrews et al. 1999, page 497; Berndt 2006, page 10] says

$$
\prod_{n=1}^{\infty}\left(1-q^{n}\right)\left(1-z q^{n-1}\right)\left(1-q^{n} / z\right)=\sum_{n=-\infty}^{\infty}(-1)^{n} q^{n(n-1) / 2} z^{n},
$$

and implies this:

Proposition 1.2. The infinite product expressions for $\theta_{1}, \theta_{2}, \theta_{3}$, and $\theta_{4}$ are

$$
\begin{aligned}
& \theta_{1}(z \mid \tau)=2 q^{1 / 8} \sin z \prod_{n=1}^{\infty}\left(1-q^{n}\right)\left(1-q^{n} e^{2 i z}\right)\left(1-q^{n} e^{-2 i z}\right), \\
& \theta_{2}(z \mid \tau)=2 q^{1 / 8} \cos z \prod_{n=1}^{\infty}\left(1-q^{n}\right)\left(1+q^{n} e^{2 i z}\right)\left(1+q^{n} e^{-2 i z}\right), \\
& \theta_{3}(z \mid \tau)=\prod_{n=1}^{\infty}\left(1-q^{n}\right)\left(1+q^{(n-1 / 2)} e^{2 i z}\right)\left(1+q^{(n-1 / 2)} e^{-2 i z}\right), \\
& \theta_{4}(z \mid \tau)=\prod_{n=1}^{\infty}\left(1-q^{n}\right)\left(1-q^{(n-1 / 2)} e^{2 i z}\right)\left(1-q^{(n-1 / 2)} e^{-2 i z}\right) .
\end{aligned}
$$

Let prime denote partial differentiation with respect to $z$. Then it is obvious from the infinite product representation of $\theta_{1}$ that

$$
\theta_{1}^{\prime}(0 \mid \tau)=2 q^{1 / 8} \prod_{n=1}^{\infty}\left(1-q^{n}\right)^{3}=2 \eta^{3}(\tau),
$$

where $\eta(\tau)$ is the well-known Dedekind $\eta$-function defined as

$$
\eta(\tau)=q^{1 / 24} \prod_{n=1}^{\infty}\left(1-q^{n}\right) .
$$

In this paper we also need modular functions $\lambda, \mu$, and $v$, which are given by

$$
\lambda(\tau)=\frac{\eta(\tau)}{\eta^{7}(\tau)}, \quad \mu(\tau)=\frac{\eta^{4}(\tau)}{\eta^{4}(7 \tau)}, \quad \text { and } \quad \nu(\tau)=\frac{\eta(\tau / 7)}{\eta(\tau)} .
$$

Ramanujan's theta functions $\phi(q)$ and $\psi(q)$ are defined as

$$
\phi(q)=\sum_{n=-\infty}^{\infty} q^{n^{2}} \text { and } \psi(q)=\sum_{n=0}^{\infty} q^{n(n+1) / 2} .
$$

It is obvious that $\phi(q)=\theta_{3}(0 \mid 2 \tau)$ and $2 \psi(q)=q^{-1 / 8} \theta_{2}(0 \mid \tau)$. Using the infinite product representations of $\theta_{2}$ and $\theta_{3}$, we can easily deduce the Gauss identities for 
$\phi(-q)$ and $\psi(q)$ :

$$
\phi(-q)=\prod_{n=1}^{\infty} \frac{\left(1-q^{n}\right)}{\left(1+q^{n}\right)} \quad \text { and } \quad \psi(q)=\prod_{n=1}^{\infty} \frac{\left(1-q^{2 n}\right)}{\left(1-q^{2 n-1}\right)} .
$$

In this paper we prove the following new theta function identity using the theory of elliptic theta functions, and discuss many important applications of this identity. Theorem 1.3. Let $f(z)$ be an entire function satisfying the functional equations

$$
f(z)=f(z+\pi)=q^{2} e^{8 i z} f(z+\pi \tau) .
$$

Then for any two complex numbers $x$ and $y$, we have the identity

$$
\begin{aligned}
& f(-x-y) \theta_{1}(x \mid \tau) \theta_{1}(y \mid \tau) \theta_{1}(x-y \mid \tau) \\
& =\quad f(x) \theta_{1}(y \mid \tau) \theta_{1}(x+2 y \mid \tau) \theta_{1}(x+y \mid \tau) \\
& -f(y) \theta_{1}(x \mid \tau) \theta_{1}(2 x+y \mid \tau) \theta_{1}(x+y \mid \tau) \\
& \quad+f(0) \theta_{1}(x-y \mid \tau) \theta_{1}(2 x+y \mid \tau) \theta_{1}(x+2 y \mid \tau) .
\end{aligned}
$$

If $h(z)$ is an entire function that satisfies the functional equations

$$
h(z)=-h(z+\pi)=-q^{3 / 2} e^{6 i z} h(z+\pi \tau),
$$

then it is easy to see that $h(z) \theta_{1}(z \mid \tau)$ satisfies the functional equations (1-6). Thus we can choose $f(z)=h(z) \theta_{1}(z \mid \tau)$ in Theorem 1.3. Using the obvious fact $\theta_{1}(0 \mid \tau)=0$ and simplifying, we deduce the following corollary.

Corollary 1.4. Let $h(z)$ be an entire function satisfying the functional equations (1-7). Then

$$
h(x) \theta_{1}(x+2 y \mid \tau)-h(y) \theta_{1}(2 x+y \mid \tau)+h(-x-y) \theta_{1}(x-y \mid \tau)=0 .
$$

Taking $y=0$ in this equation and then replacing $x$ by $z$, we immediately find a general quintuple product identity [Liu 2005]:

Corollary 1.5. Let $h(z)$ be an entire function satisfying the functional equations (1-7). Then

$$
h(z)+h(-z)=h(0) \frac{\theta_{1}(2 z \mid \tau)}{\theta_{1}(z \mid \tau)} .
$$

Differentiate the identity in Theorem 1.3 with respect to $x$, set $x=0$, and then write $y$ as $z$ to obtain the following amusing identity, which is equivalent to the main result of [Liu 2005, Theorem 1] and has many applications.

Corollary 1.6. Let $f(z)$ be an entire function satisfying the functional equations in Theorem 1.3. Then

$$
f(z)-f(-z)=\frac{f^{\prime}(0)}{\theta_{1}^{\prime}(0 \mid \tau)} \theta_{1}(2 z \mid \tau) .
$$


The rest of this paper is organized as follows. In Section 2 we prove Theorem 1.3 with the theory of the elliptic theta functions. In Section 3, we use this theorem to give a new derivation of the following Eisenstein series identity of Ramanujan [Berndt 1991, (5.15)].

Lemma 1.7. Let $\phi(q)$ and $\psi(q)$ be the Ramanujan theta functions as in (1-4), and let $\left(\frac{n}{7}\right)$ be the Legendre symbol. Then

$$
1+2 \sum_{n=1}^{\infty}\left(\frac{n}{7}\right) \frac{q^{n}}{1-q^{n}}=4 q^{2} \psi(q) \psi\left(q^{14}\right)+\phi(q) \phi\left(q^{7}\right) .
$$

In Section 4, we use Theorem 1.3 to prove the following result of Ramanujan; see [Berndt 1991, first identity in entry 5(i) of Chapter 21].

Lemma 1.8 [Berndt 1991, page 467]. Let $\left(\frac{n}{7}\right)$ be the Legendre symbol. Then

$$
\left(1+2 \sum_{n=1}^{\infty}\left(\frac{n}{7}\right) \frac{q^{n}}{1-q^{n}}\right)^{2}=1+4 \sum_{n=1}^{\infty} \frac{n q^{n}}{1-q^{n}}-28 \sum_{n=1}^{\infty} \frac{n q^{7 n}}{1-q^{7 n}} .
$$

Following Ramanujan and [Berndt 1991], we define modular functions $\alpha, \beta$ and $t$, and $x_{1}$ and $x_{2}$ as

$$
\begin{aligned}
\alpha & =\theta_{2}^{4}(0 \mid \tau) / \theta_{3}^{4}(0 \mid \tau), & \beta & =\theta_{2}^{4}(0 \mid 7 \tau) / \theta_{3}^{4}(0 \mid 7 \tau), \\
x_{1} & =(\alpha(1-\alpha) / t(1-t))^{1 / 3}, & x_{2} & =(\beta(1-\beta) / t(1-t))^{1 / 3} .
\end{aligned}
$$

In Section 5, we provide a simple proof of the following lemma, which gives us a quadratic equation whose solutions are modular functions.

Lemma 1.9. Let $t, x_{1}$ and $x_{2}$ be defined as in (1-10). Then $x_{1}$ and $x_{2}$ are the roots of the quadratic equation

$$
x^{2}-\left(2-7 t+11 t^{2}-8 t^{3}+4 t^{4}\right) x+t^{2}(1-t)^{2}=0,
$$

and we have

$$
\begin{aligned}
& 2 x_{1}=2-7 t+11 t^{2}-8 t^{3}+4 t^{4}+(1-2 t) R, \\
& 2 x_{2}=2-7 t+11 t^{2}-8 t^{3}+4 t^{4}-(1-2 t) R,
\end{aligned}
$$

where

$$
R=\sqrt{\left(2-3 t+2 t^{2}\right)\left(2-t+t^{2}\right)\left(1-t+2 t^{2}\right)} .
$$

In Section 6, we apply Lemmas 1.7, 1.8, and 1.9 and the Jacobi four-square formula to provide a new proof of the following identity. 
Lemma 1.10. Suppose $m=\theta_{3}^{2}(0 \mid \tau) / \theta_{3}^{2}(0 \mid 7 \tau)$, and let $t$ be defined as in (1-8). Then

$$
m-7 / m=-6+16 t-12 t^{2}+8 t^{3} .
$$

Let $R$ be defined as in (1-12). We further have

$$
\begin{aligned}
m & =-3+8 t-6 t^{2}+4 t^{3}+2 R, \\
-7 / m & =-3+8 t-6 t^{2}=4 t^{3}-2 R .
\end{aligned}
$$

In Section 7, we use Lemmas 1.7, 1.8, 1.9, and 1.10 to prove the following remarkable identity of Ramanujan, which expresses an Eisenstein series in terms of the $\eta$-function.

Theorem 1.11. Let $\lambda$ and $\mu$ be defined as in (1-3). Then

$$
\left(1+2 \sum_{n=1}^{\infty}\left(\frac{n}{7}\right) \frac{q^{n}}{1-q^{n}}\right)^{3}=\lambda^{-1}\left(\mu^{2}+13 \mu+49\right) .
$$

In Section 8, we use Theorems 1.3 and 1.11 and the theory of elliptic functions to give a new proof of the following Ramanujan-Watson modular equation of the seventh order, which plays a pivotal role in the study of the theory of modular equations of degree 7. For other proofs, see [Berndt 1991, pages 306-311; Fine 1956; Lachaud 2005; Watson 1938].

Theorem 1.12 (Ramanujan and Watson). With $\mu$ and $v$ defined as in (1-3), we have

$$
2 \mu=7\left(v^{3}+5 v^{2}+7 v\right)+\left(v^{2}+7 v+7\right) \sqrt{4 v^{3}+21 v^{2}+28 v} .
$$

In Sections 9 and 10 we derive some theta function identities related to modular equations of degree 7 and some curious finite trigonometric sums. For example,

$$
\left(\frac{\sin (2 \pi / 7)}{\sin (\pi / 7)}\right)^{14}+\left(\frac{\sin (3 \pi / 7)}{\sin (2 \pi / 7)}\right)^{14}+\left(\frac{\sin (\pi / 7)}{\sin (3 \pi / 7)}\right)^{14}=3827
$$

Definition 1.13. The Bernoulli numbers $B_{k}$ are defined as the coefficients in the power series

$$
\frac{z}{e^{z}-1}=\sum_{k=0}^{\infty} B_{k} \frac{z^{k}}{k !} \quad \text { for }|z|<2 \pi .
$$

It is easy to show that $B_{2 k+1}=0$ for $k \geq 1$, and that the first few nonzero values of $B_{k}$ are

$$
\begin{array}{llll}
B_{0}=1, & B_{1}=-\frac{1}{2}, & B_{2}=\frac{1}{6}, & B_{4}=-\frac{1}{30}, \\
B_{6}=\frac{1}{42}, & B_{8}=-\frac{1}{30}, & B_{10}=\frac{5}{66}, & B_{12}=-\frac{691}{2730} .
\end{array}
$$


Definition 1.14. The normalized Eisenstein series $E_{2 k}$ is defined as

$$
E_{2 k}(\tau)=1-\frac{4 k}{B_{2 k}} \sum_{n=1}^{\infty} \frac{n^{2 k-1} q^{n}}{1-q^{n}} .
$$

It is easily seen that the first three Eisenstein series are given by

$$
\begin{aligned}
& E_{2}(\tau)=1-24 \sum_{n=1}^{\infty} \frac{n q^{n}}{1-q^{n}}, \quad E_{4}(\tau)=1+240 \sum_{n=1}^{\infty} \frac{n^{3} q^{n}}{1-q^{n}}, \\
& E_{6}(\tau)=1-504 \sum_{n=1}^{\infty} \frac{n^{3} q^{n}}{1-q^{n}} .
\end{aligned}
$$

In this paper we use $J_{k}(z \mid \tau)$ to denote the logarithmic derivative of $\theta_{k}$ with respect to the variable $z$ for $k=1,2,3,4$.

In Section 11, we use the general quintuple identity in Corollary 1.5 to derive the following two new and nontrivial elliptic theta function identities with the help of logarithmic differentiation.

Theorem 1.15. If $J_{1}$ denotes the logarithmic derivative of $\theta_{1}$ with respect to $z$, then

$$
\begin{aligned}
& 2\left(J_{1}(x \mid \tau)+J_{1}(y \mid \tau)-J_{1}(x+y \mid \tau)\right)^{4} \\
& -4\left(J_{1}(x \mid \tau)+J_{1}(y \mid \tau)-J_{1}(x+y \mid \tau)\right) \times\left(J_{1}^{\prime \prime}(x \mid \tau)+J_{1}^{\prime \prime}(y \mid \tau)-J_{1}^{\prime \prime}(x+y \mid \tau)\right) \\
& =2 E_{4}(\tau)+J_{1}^{\prime \prime \prime}(x \mid \tau)+J_{1}^{\prime \prime \prime}(y \mid \tau)+J_{1}^{\prime \prime \prime}(x+y \mid \tau) .
\end{aligned}
$$

Theorem 1.16. If $J_{1}$ denotes the logarithmic derivative of $\theta_{1}$ with respect to $z$, then

$$
\begin{array}{r}
40\left(J_{1}(x \mid \tau)+J_{1}(y \mid \tau)-J_{1}(x+y \mid \tau)\right)^{3} \times\left(J_{1}^{\prime \prime}(x \mid \tau)+J_{1}^{\prime \prime}(y \mid \tau)-J_{1}^{\prime \prime}(x+y \mid \tau)\right) \\
-6\left(J_{1}(x \mid \tau)+J_{1}(y \mid \tau)-J_{1}(x+y \mid \tau)\right) \times\left(J_{1}^{(4)}(x \mid \tau)+J_{1}^{(4)}(y \mid \tau)-J_{1}^{(4)}(x+y \mid \tau)\right) \\
-10\left(J_{1}^{\prime \prime}(x \mid \tau)+J_{1}^{\prime \prime}(y \mid \tau)-J_{1}^{\prime \prime}(x+y \mid \tau)\right)^{2}-16\left(J_{1}(x \mid \tau)+J_{1}(y \mid \tau)-J_{1}(x+y \mid \tau)\right)^{6} \\
=16 E_{6}(\tau)+J_{1}^{(5)}(x \mid \tau)+J_{1}^{(5)}(y \mid \tau)+J_{1}^{(5)}(x+y \mid \tau) .
\end{array}
$$

In Section 12, Theorems 1.15 and 1.16 will be used to derive the following two Eisenstein series identities of Ramanujan related to modular equations of degree 7 [Ramanujan 1988, page 53]; different proofs of these two identities can be found in [Berndt et al. 2000; Liu 2003; Raghavan and Rangachari 1989].

Theorem 1.17. Let $\mu, v$ be defined as in (1-3). Then we have

$$
\begin{aligned}
E_{4}(\tau) & =\lambda^{-4 / 3}\left(\mu^{2}+5 \times 7^{2} \mu+7^{4}\right)\left(\mu^{2}+13 \mu+49\right)^{1 / 3}, \\
E_{4}(7 \tau) & =\lambda^{-4 / 3}\left(\mu^{2}+5 \mu+1\right)\left(\mu^{2}+13 \mu+49\right)^{1 / 3}, \\
E_{6}(\tau) & =\lambda^{-2}\left(\mu^{4}-10 \times 7^{2} \mu^{3}-9 \times \mu^{2}-2 \times 7^{6} \mu-7^{7}\right), \\
E_{6}(7 \tau) & =\lambda^{-2}\left(\mu^{4}+14 \mu^{3}+63 \mu^{2}+70 \mu-7\right) .
\end{aligned}
$$


As shown by Raghavan and Rangachari [1989], these identities are equivalent to Klein's formulas [1999], which express the modular $j$-invariant in terms of $\mu$.

In Section 13, Theorems 1.15 and 1.16 are used to derive the following two remarkable Lambert series identities.

\section{Theorem 1.18.}

$$
\begin{aligned}
& \left(\cot z-4 \sum_{n=1}^{\infty} \frac{q^{n}}{1+q^{n}} \sin 2 n z\right)^{4} \\
& =4\left(\cot z-4 \sum_{n=1}^{\infty} \frac{q^{n}}{1+q^{n}} \sin 2 n z\right)\left(\cot z+\cot ^{2} z+8 \sum_{n=1}^{\infty} \frac{n^{2} q^{n}}{1+q^{n}} \sin 2 n z\right) \\
& \quad-\cot ^{2} z\left(4+3 \cot ^{2} z\right)-16 \sum_{n=1}^{\infty} \frac{n^{3} q^{n}}{1-q^{n}} \cos 2 n z+16 \sum_{n=1}^{\infty}(-1)^{n} \frac{n^{3} q^{n}}{1-q^{n}} .
\end{aligned}
$$

\section{Theorem 1.19.}

$$
\begin{aligned}
&\left(\csc z+4 \sum_{n=0}^{\infty} \frac{q^{2 n+1}}{1-q^{2 n+1}} \sin (2 n+1) z\right)^{4} \\
&=2\left(\csc z+4 \sum_{n=0}^{\infty} \frac{q^{2 n+1}}{1-q^{2 n+1}} \sin (2 n+1) z\right) \\
& \quad \times\left(2 \csc ^{3} z-\csc z-4 \sum_{n=0}^{\infty} \frac{(2 n+1)^{2} q^{2 n+1}}{1-q^{2 n+1}} \sin (2 n+1) z\right) \\
& \quad+2 \csc ^{2} z-3 \csc ^{4} z-16 \sum_{n=1}^{\infty} \frac{n^{3} q^{2 n}}{1-q^{2 n}} \cos 2 n z+16 \sum_{n=1}^{\infty} \frac{n^{3} q^{n}}{1-q^{2 n}}
\end{aligned}
$$

Finally, in Section 14 we establish the following identity.

Theorem 1.20. Let $\mu, v$ be defined as in (1-3). Then we have

$$
\frac{\theta_{2}^{3}(0 \mid \tau)}{\theta_{2}(0 \mid 7 \tau)}-\frac{\theta_{3}^{3}(0 \mid \tau)}{\theta_{3}(0 \mid 7 \tau)}+\frac{\theta_{4}^{3}(0 \mid \tau)}{\theta_{4}(0 \mid 7 \tau)}=4 \mu^{1 / 2} \lambda^{-1 / 3}\left(\mu^{2}+13 \mu+49\right)^{1 / 3} .
$$

\section{The proof of Theorem 1.3}

We prove Theorem 1.3 with the complex theory of elliptic theta functions. Proof. It is easy to show directly that $\theta_{1}$ satisfies the functional equations

$$
\theta_{1}(z \mid \tau)=-\theta_{1}(z+\pi \mid \tau)=-q^{1 / 2} e^{2 i z} \theta_{1}(z+\pi \tau \mid \tau) .
$$


Suppose that $f(z)$ satisfies the functional equations in Theorem 1.3. Now we consider the function $g(z)$ given by

$$
g(z)=\frac{f(z)}{\theta_{1}(z \mid \tau) \theta_{1}(z-x \mid \tau) \theta_{1}(z-y \mid \tau) \theta_{1}(z+x+y \mid \tau)},
$$

where we temporarily assume that $0<x, y, x+y<\pi$. Using (2-1) we can verify that $g(z)=g(z+\pi)=g(z+\pi \tau)$. Hence $g(z)$ is an elliptic function with periods $\pi$ and $\pi \tau$. It is obvious that $0, x, y$ and $\pi-x-y$ are the only poles of $g(z)$ and that all poles are simple. We will use $\operatorname{res}(g ; x)$ to denote the residue of $g$ at $\alpha$. It is well known that the sum of all residues of an elliptic function at the poles inside any cell is zero (see, for example [Apostol 1990, page 6]). So we have

$$
\operatorname{res}(g ; 0)+\operatorname{res}(g ; x)+\operatorname{res}(g ; y)+\operatorname{res}(g ; \pi-x-y)=0 .
$$

By direct and elementary computations, we have

$$
\begin{gathered}
\operatorname{res}(g ; 0)=\frac{f(0)}{\theta_{1}^{\prime}(0 \mid \tau) \theta_{1}(x \mid \tau) \theta_{1}(y \mid \tau) \theta_{1}(x+y \mid \tau)}, \\
\operatorname{res}(g ; x)=\frac{f(x)}{\theta_{1}^{\prime}(0 \mid \tau) \theta_{1}(x \mid \tau) \theta_{1}(x-y \mid \tau) \theta_{1}(2 x+y \mid \tau)}, \\
\operatorname{res}(g ; y)=-\frac{f(y)}{\theta_{1}^{\prime}(0 \mid \tau) \theta_{1}(y \mid \tau) \theta_{1}(x-y \mid \tau) \theta_{1}(x+2 y \mid \tau)}, \\
\operatorname{res}(g ; \pi-x-y)=-\frac{f(-x-y)}{\theta_{1}^{\prime}(0 \mid \tau) \theta_{1}(x+y \mid \tau) \theta_{1}(2 x+y \mid \tau) \theta_{1}(x+2 y \mid \tau)} .
\end{gathered}
$$

Substituting the equations above into (2-2) and simplifying, we arrive at the identity in Theorem 1.3. By analytic continuation we know the identity in Theorem 1.3 holds for any $x$ and $y$, and this completes the proof.

The four Jacobi theta functions are mutually related, and starting from one of them we may obtain the other three by simple calculations. For example, we have the following propositions.

Proposition 2.1. The Jacobi theta functions $\theta_{1}, \theta_{2}, \theta_{3}$, and $\theta_{4}$ satisfy the relations

$$
\begin{aligned}
\theta_{1}(z+\pi / 2 \mid \tau) & =\theta_{2}(z \mid \tau), \\
\theta_{1}(z+(\pi \tau) / 2 \mid \tau) & =i q^{-1 / 8} e^{-i z} \theta_{4}(z \mid \tau), \\
\theta_{1}(z+(\pi+\pi \tau) / 2 \mid \tau) & =q^{-1 / 8} e^{-i z} \theta_{3}(z \mid \tau) .
\end{aligned}
$$

Corollary 2.2. Let $f(z)$ be an entire function satisfying the functional equations in Theorem 1.3. Then

$$
f\left(\frac{1}{2} \pi\right)+q^{1 / 2} f\left(\frac{1}{2} \pi \tau\right)=f(0)+q^{1 / 2} f\left(\frac{1}{2}(\pi+\pi \tau)\right) .
$$


Proof. We take $x=\pi / 2$ and $y=(\pi \tau) / 2$ in Theorem 1.3 and then use (2-1) and Proposition 2.1 to simplify the resulting equation.

It is easy to check that $\theta_{1}^{4}(z \mid \tau)$ is an entire function of $z$ and satisfies the functional equations in Theorem 1.3. So we can take $f(z)=\theta_{1}^{4}(z \mid \tau)$ in Corollary 2.2 and immediately deduce the Jacobi quartic identity

$$
\theta_{2}^{4}(0 \mid \tau)+\theta_{4}^{4}(0 \mid \tau)=\theta_{3}^{4}(0 \mid \tau) .
$$

It is easy to verify that the product

$$
f(z)=\theta_{1}(z \mid \tau) \theta_{1}(z+x \mid \tau) \theta_{1}(z+y \mid \tau) \theta_{1}(z-x-y \mid \tau) .
$$

of theta functions satisfies the conditions of Corollary 2.2. Thus we can substitute this function into Corollary 2.2 and find that

$$
\begin{gathered}
\theta_{3}(0 \mid \tau) \theta_{3}(x \mid \tau) \theta_{3}(y \mid \tau) \theta_{3}(x+y \mid \tau) \\
-\theta_{2}(0 \mid \tau) \theta_{2}(x \mid \tau) \theta_{2}(y \mid \tau) \theta_{2}(x+y \mid \tau) \\
-\theta_{4}(0 \mid \tau) \theta_{4}(x \mid \tau) \theta_{4}(y \mid \tau) \theta_{4}(x+y \mid \tau)=0
\end{gathered}
$$

Corollary 2.3 [Berndt 1991, entry 19(i); Guetzlaff 1834].

$$
\sqrt{\theta_{2}(0 \mid \tau) \theta_{2}(0 \mid 7 \tau)}+\sqrt{\theta_{4}(0 \mid \tau) \theta_{4}(0 \mid 7 \tau)}=\sqrt{\theta_{3}(0 \mid \tau) \theta_{3}(0 \mid 7 \tau)} .
$$

Proof. Replace $\tau$ with $7 \tau$ and then take $x=\pi \tau$ and $y=2 \pi \tau$ in the resulting equation to deduce that

$$
\begin{gathered}
\theta_{3}(0 \mid 7 \tau) \theta_{3}(\pi \tau \mid 7 \tau) \theta_{3}(2 \pi \tau \mid 7 \tau) \theta_{3}(3 \pi \tau \mid 7 \tau) \\
-\theta_{2}(0 \mid 7 \tau) \theta_{2}(\pi \tau \mid 7 \tau) \theta_{2}(2 \pi \tau \mid 7 \tau) \theta_{2}(3 \pi \tau \mid 7 \tau) \\
-\theta_{4}(0 \mid 7 \tau) \theta_{4}(\pi \tau \mid 7 \tau) \theta_{4}(2 \pi \tau \mid 7 \tau) \theta_{4}(3 \pi \tau \mid 7 \tau)=0
\end{gathered}
$$

Employing the infinite product representations of theta functions, we may find for $k \in\{2,3,4\}$ that

$$
\theta_{k}(\pi \tau \mid 7 \tau) \theta_{k}(2 \pi \tau \mid 7 \tau) \theta_{k}(3 \pi \tau \mid 7 \tau)=\sqrt{\prod_{n=1}^{\infty} \frac{\left(1-q^{7 n}\right)^{7}}{\left(1-q^{n}\right)} \frac{\theta_{k}(0 \mid \tau)}{\theta_{k}(0 \mid 7 \tau)}}
$$

Substituting the above into (2-4) and simplifying, we immediately arrive at the Guetzlaff identity.

Next we give more applications of Corollary 2.2. Taking

$$
f(z)=\theta_{1}(z+u \mid \tau) \theta_{1}(z+v \mid \tau) \theta_{1}(z+w \mid \tau) \theta_{1}(z-u-v-w \mid \tau),
$$


we deduce the identity [Liu 2001]

$$
\begin{aligned}
& \theta_{1}(u \mid \tau) \theta_{1}(v \mid \tau) \theta_{1}(w \mid \tau) \theta_{1}(u+v+w \mid \tau) \\
+ & \theta_{2}(u \mid \tau) \theta_{2}(v \mid \tau) \theta_{2}(w \mid \tau) \theta_{2}(u+v+w \mid \tau) \\
+ & \theta_{4}(u \mid \tau) \theta_{4}(v \mid \tau) \theta_{4}(w \mid \tau) \theta_{4}(u+v+w \mid \tau) \\
- & \theta_{3}(u \mid \tau) \theta_{3}(v \mid \tau) \theta_{3}(w \mid \tau) \theta_{3}(u+v+w \mid \tau)=0
\end{aligned}
$$

Letting $f(z)=\theta_{1}(z \mid \tau) \theta_{1}(3 z \mid 3 \tau)$, we conclude that [Berndt 1991, entry 5(ii)]

$$
\theta_{2}(0 \mid \tau) \theta_{2}(0 \mid 3 \tau)+\theta_{4}(0 \mid \tau) \theta_{4}(0 \mid 3 \tau)=\theta_{3}(0 \mid \tau) \theta_{3}(0 \mid 3 \tau) .
$$

Taking $f(z)=\theta_{1}(z \mid \tau) \theta_{1}^{3}(z+\pi / 3 \mid \tau)$ in Corollary 2.2 and simplifying, we find that

$$
\sqrt{\frac{\theta_{2}^{3}(0 \mid 3 \tau)}{\theta_{2}(0 \mid \tau)}}=\sqrt{\frac{\theta_{4}^{3}(0 \mid 3 \tau)}{\theta_{4}(0 \mid \tau)}}-\sqrt{\frac{\theta_{3}^{3}(0 \mid 3 \tau)}{\theta_{3}(0 \mid \tau)}} .
$$

\section{The proof of Lemma 1.7}

In this section we use Corollary 1.5 to prove Lemma 1.7.

Proof. Replacing $z$ with $z+\pi / 2$ in Corollary 1.5 and simplifying, we find that

$$
h(\pi / 2+z)+h(-\pi / 2-z)=-h(0) \frac{\theta_{1}(2 z \mid \tau)}{\theta_{2}(z \mid \tau)} .
$$

If we differentiate this equation with respect to $z$ and let $z \rightarrow 0$, we deduce that

$$
h^{\prime}(\pi / 2)-h^{\prime}(-\pi / 2)=-2 h(0) \frac{\theta_{1}^{\prime}(0 \mid \tau)}{\theta_{2}(0 \mid \tau)} .
$$

We choose $h(z)=\theta_{1}(z+x \mid \tau) \theta_{1}(z+y \mid \tau) \theta_{1}(z-x-y \mid \tau)$ and observe that

$$
\theta_{1}(z+\pi / 2 \mid \tau)=\theta_{2}(z \mid \tau)
$$

Then (3-1) becomes

$$
J_{2}(x \mid \tau)+J_{2}(y \mid \tau)-J_{2}(x+y \mid \tau)=\frac{\theta_{1}^{\prime}(0 \mid \tau) \theta_{1}(x \mid \tau) \theta_{1}(y \mid \tau) \theta_{1}(x+y \mid \tau)}{\theta_{2}(0 \mid \tau) \theta_{2}(x \mid \tau) \theta_{2}(y \mid \tau) \theta_{2}(x+y \mid \tau)} .
$$

The trigonometric series expansion for $J_{2}(z \mid \tau)$ (see for example [Whittaker and Watson 1996, page 489]) is

$$
J_{2}(z \mid \tau)=-\tan z+4 \sum_{n=1}^{\infty} \frac{(-q)^{n}}{1-q^{n}} \sin 2 n z .
$$


Substituting this into (3-2), we find an identity involving trigonometric series and theta functions:

$$
\begin{array}{r}
-\tan x-\tan y+\tan (x+y)+4 \sum_{n=1}^{\infty} \frac{(-q)^{n}}{1-q^{n}}(\sin 2 n x+\sin 2 n y-\sin 2 n(x+y)) \\
=\frac{\theta_{1}^{\prime}(0 \mid \tau) \theta_{1}(x \mid \tau) \theta_{1}(y \mid \tau) \theta_{1}(x+y \mid \tau)}{\theta_{2}(0 \mid \tau) \theta_{2}(x \mid \tau) \theta_{2}(y \mid \tau) \theta_{2}(x+y \mid \tau)} .
\end{array}
$$

Take $x=\pi / 7$ and $y=2 \pi / 7$ in this equation to deduce that

$$
C+4 \sum_{n=1}^{\infty} \frac{(-q)^{n}}{1-q^{n}} T(n)=\frac{\theta_{1}^{\prime}(0 \mid \tau) \theta_{1}(\pi / 7 \mid \tau) \theta_{1}(2 \pi / 7 \mid \tau) \theta_{1}(3 \pi / 7 \mid \tau)}{\theta_{2}(0 \mid \tau) \theta_{2}(\pi / 7 \mid \tau) \theta_{2}(2 \pi / 7 \mid \tau) \theta_{2}(3 \pi / 7 \mid \tau)}
$$

where

$$
C=-\tan \frac{\pi}{7}-\tan \frac{2 \pi}{7}+\tan \frac{3 \pi}{7} \quad \text { and } \quad T(n)=\sin \frac{2 n \pi}{7}+\sin \frac{4 n \pi}{7}-\sin \frac{6 n \pi}{7} .
$$

Using the infinite product expansions for $\theta_{1}$ and $\theta_{2}$, we can readily find that

$$
\begin{aligned}
& \theta_{1}\left(\frac{\pi}{7} \mid \tau\right) \theta_{1}\left(\frac{2 \pi}{7} \mid \tau\right) \theta_{1}\left(\frac{3 \pi}{7} \mid \tau\right)=\sqrt{7} q^{3 / 8} \prod_{n=1}^{\infty}\left(1-q^{n}\right)^{2}\left(1-q^{7 n}\right), \\
& \theta_{2}\left(\frac{\pi}{7} \mid \tau\right) \theta_{2}\left(\frac{2 \pi}{7} \mid \tau\right) \theta_{2}\left(\frac{3 \pi}{7} \mid \tau\right)=q^{3 / 8} \prod_{n=1}^{\infty}\left(1+q^{7 n}\right) \frac{\left(1-q^{n}\right)^{3}}{\left(1+q^{n}\right)} .
\end{aligned}
$$

Substituting these two identities into (3-5) and then using the Gauss identity for $\phi(-q)$ in (1-5) gives

$$
C+4 \sum_{n=1}^{\infty} \frac{(-q)^{n}}{1-q^{n}} T(n)=\sqrt{7} \phi(-q) \phi\left(-q^{7}\right) .
$$

To simplify the left side of this equation, we should compute $C$ and $T(n)$. Fortunately, we don't need to find these values from other sources, as we can determine them from (3-6). By equating the constant term, we obtain $C=\sqrt{7}$, and by equating the coefficients of $q$ and then using the properties of the sine function we readily find that

$$
T(n)=\sin \frac{2 n \pi}{7}+\sin \frac{4 n \pi}{7}-\sin \frac{6 n \pi}{7}=\frac{\sqrt{7}}{2}\left(\frac{n}{7}\right) .
$$

Thus we conclude that

$$
1+2 \sum_{n=1}^{\infty}\left(\frac{n}{7}\right) \frac{(-q)^{n}}{1-q^{n}}=\phi(-q) \phi\left(-q^{7}\right) .
$$


Replacing $q$ by $-q$ in this equation will yield [Berndt 1991, page 302, entry 17(ii)]

$$
1+2 \sum_{n=1}^{\infty}\left(\frac{n}{7}\right) \frac{q^{n}}{1-(-q)^{n}}=\phi(q) \phi\left(q^{7}\right) .
$$

Replace $z$ with $z+\pi \tau / 2$ in Corollary 1.5 and simplify to deduce that

$$
h\left(\frac{1}{2} \pi \tau+z\right)+h\left(-\frac{1}{2} \pi \tau-z\right)=i q^{-3 / 8} h(0) e^{-3 i z} \frac{\theta_{1}(2 z \mid \tau)}{\theta_{4}(z \mid \tau)} .
$$

If we differentiate this equation with respect to $z$ and let $z \rightarrow 0$, we find that

$$
h^{\prime}\left(\frac{1}{2} \pi \tau\right)-h^{\prime}\left(-\frac{1}{2} \pi \tau\right)=2 i q^{-3 / 8} h(0) \frac{\theta_{1}^{\prime}(0 \mid \tau)}{\theta_{4}(0 \mid \tau)} .
$$

Letting $h(z)=\theta_{1}(z+x \mid \tau) \theta_{1}(z+y \mid \tau) \theta_{1}(z-x-y \mid \tau)$ in the above, we find that [Whittaker and Watson 1996, page 490]

(3-9) $J_{4}(x \mid \tau)+J_{4}(y \mid \tau)-J_{4}(x+y \mid \tau)=\frac{\theta_{1}^{\prime}(0 \mid \tau) \theta_{1}(x \mid \tau) \theta_{1}(y \mid \tau) \theta_{1}(x+y \mid \tau)}{\theta_{4}(0 \mid \tau) \theta_{4}(x \mid \tau) \theta_{4}(y \mid \tau) \theta_{4}(x+y \mid \tau)}$.

The trigonometric series expansion for $J_{4}(z \mid \tau)$ (see for example [Whittaker and Watson 1996, page 489]) states that

$$
J_{4}(z \mid \tau)=4 \sum_{n=1}^{\infty} \frac{q^{n / 2}}{1-q^{n}} \sin 2 n z .
$$

Substituting the equation above into (3-9), we immediately find that

$$
\begin{aligned}
4 \sum_{n=1}^{\infty} \frac{q^{n / 2}}{1-q^{n}}(\sin 2 n x+\sin 2 n y & -\sin 2 n(x+y)) \\
& =\frac{\theta_{1}^{\prime}(0 \mid \tau) \theta_{1}(x \mid \tau) \theta_{1}(y \mid \tau) \theta_{1}(x+y \mid \tau)}{\theta_{4}(0 \mid \tau) \theta_{4}(x \mid \tau) \theta_{4}(y \mid \tau) \theta_{4}(x+y \mid \tau)} .
\end{aligned}
$$

Replacing $q$ by $q^{2}$ in the equation above, then taking $x=\pi / 7$ and $y=2 \pi / 7$ in the resulting equation, and employing the same type of argument as that of deriving (3-8) from (3-5), we find that [Berndt 1991, entry 17(i)]

$$
\sum_{n=1}^{\infty}\left(\frac{n}{7}\right) \frac{q^{n}}{1-q^{2 n}}=q \psi(q) \psi\left(q^{7}\right) .
$$

Now we will begin to prove (1-8) from (3-8) and (3-12). It is obvious that

$$
\frac{2 q^{2 n}}{1-q^{4 n}}=\frac{q^{2 n}}{1-q^{2 n}}+\frac{q^{2 n}}{1+q^{2 n}} .
$$


Replacing $q$ by $q^{2}$ in (3-12) and then substituting the above into the resulting equation, we can find that

$$
4 q^{2} \psi\left(q^{2}\right) \psi\left(q^{14}\right)=2 \sum_{n=1}^{\infty}\left(\frac{n}{7}\right) \frac{q^{2 n}}{1-q^{2 n}}+2 \sum_{n=1}^{\infty}\left(\frac{n}{7}\right) \frac{q^{2 n}}{1+q^{2 n}} .
$$

Using the elementary identity $\left(1-(-q)^{n}\right)\left(1+(-q)^{n}\right)=1-q^{2 n}$ in (3-8), we obtain

$$
\phi(q) \phi\left(q^{7}\right)=1+2 \sum_{n=1}^{\infty}\left(\frac{n}{7}\right) \frac{q^{n}\left(1+(-q)^{n}\right)}{1-q^{2 n}} .
$$

Adding the last two equations together, we immediately find that

$$
\begin{aligned}
\phi(q) \phi\left(q^{7}\right) & +4 q^{2} \psi\left(q^{2}\right) \psi\left(q^{14}\right) \\
& =1+2 \sum_{n=1}^{\infty}\left(\frac{n}{7}\right) \frac{q^{n}}{1-q^{2 n}}+\sum_{n=1}^{\infty}\left(\frac{n}{7}\right) \frac{q^{2 n}}{1+q^{2 n}}+2 \sum_{n=1}^{\infty}\left(\frac{n}{7}\right) \frac{\left(1+(-1)^{n}\right) q^{2 n}}{1-q^{2 n}} .
\end{aligned}
$$

Making use of the property $\left(\frac{2 n}{7}\right)=\left(\frac{n}{7}\right)$ of the Legendre symbol, we find that

$$
\sum_{n=1}^{\infty}\left(\frac{n}{7}\right) \frac{\left(1+(-1)^{n}\right) q^{2 n}}{1-q^{2 n}}=2 \sum_{n=1}^{\infty}\left(\frac{2 n}{7}\right) \frac{q^{4 n}}{1-q^{4 n}}=2 \sum_{n=1}^{\infty}\left(\frac{n}{7}\right) \frac{q^{4 n}}{1-q^{4 n}} .
$$

Combining these equations, we find that

$$
\begin{aligned}
\phi(q) \phi\left(q^{7}\right)+4 q^{2} \psi\left(q^{2}\right) & \psi\left(q^{14}\right) \\
& =1+2 \sum_{n=1}^{\infty}\left(\frac{n}{7}\right) \frac{q^{n}}{1-q^{2 n}}+2 \sum_{n=1}^{\infty}\left(\frac{n}{7}\right)\left(\frac{q^{2 n}}{1+q^{2 n}}+\frac{2 q^{4 n}}{1-q^{4 n}}\right) \\
& =1+2 \sum_{n=1}^{\infty}\left(\frac{n}{7}\right) \frac{q^{n}}{1-q^{2 n}}+2 \sum_{n=1}^{\infty}\left(\frac{n}{7}\right) \frac{q^{2 n}}{1-q^{2 n}} \\
& =1+2 \sum_{n=1}^{\infty}\left(\frac{n}{7}\right) \frac{q^{n}}{1-q^{n}},
\end{aligned}
$$

which is Lemma 1.7.

Remark 3.1. Dividing both sides of (3-4) by $y$ and then letting $y \rightarrow 0$, we deduce that

$$
\tan ^{2} x+8 \sum_{n=1}^{\infty} \frac{n(-q)^{n}}{1-q^{n}}(1-\cos 2 n x)=\prod_{n=1}^{\infty}\left(\frac{1-q^{n}}{1+q^{n}}\right)^{4} \frac{\theta_{1}^{2}(x \mid \tau)}{\theta_{2}^{2}(x \mid \tau)}
$$


We take $x=\pi / 4$ in this equation and use $\theta_{1}(\pi / 4 \mid \tau)=\theta_{2}(\pi / 4 \mid \tau)$ in the result. Then we immediately have

$$
\phi^{4}(-q)=\prod_{n=1}^{\infty}\left(\frac{1-q^{n}}{1+q^{n}}\right)^{4}=1+8 \sum_{n=1}^{\infty} \frac{n(-q)^{n}}{1-q^{n}}-16 \sum_{n=1}^{\infty}(-1)^{n} \frac{n q^{2 n}}{1-q^{2 n}} .
$$

Replacing $q$ by $-q$ and after simple reduction, we get the well-known Jacobi foursquares formula [Andrews et al. 1999, (10.6.7); Andrews et al. 2001; Berndt 2006, (3.3.6); Hardy and Wright 1979, Theorem 385; Hirschhorn 1987; Milne 2002].

$$
\phi^{4}(q)=1+8 \sum_{n=1}^{\infty} \frac{n q^{n}}{1-q^{n}}-32 \sum_{n=1}^{\infty} \frac{n q^{4 n}}{1-q^{4 n}} .
$$

Divide (3-11) by $x^{2}$, let $x \rightarrow 0$ and replace $q$ with $-q$. We find the Jacobi eightsquares formula (see for example [Berndt 2006, page 70])

$$
\phi^{8}(q)=1+16 \sum_{n=1}^{\infty} \frac{n^{3} q^{n}}{1-(-q)^{n}} .
$$

\section{The proof of Lemma 1.8}

Putting $h(z)=e^{2 i z} \theta_{1}(3 z+\pi \tau \mid 3 \tau)$ in Corollary 1.5 and simplifying, we can get the quintuple product identity [Liu 2005]

$$
\sum_{n=-\infty}^{\infty}(-1)^{n} q^{n(3 n+1) / 2} \cos (2 n+1) z=(q ; q)_{\infty} \frac{\theta_{1}(2 z \mid \tau)}{\theta_{1}(z \mid \tau)} .
$$

This is an important identity with a very rich history, and there are many different proofs of it in the literature. One may consult [Berndt 1991, page 83] and the survey paper [Cooper 2006] for the various proofs of this identity.

Substituting (4-1) into the right side of the identity in Corollary 1.5, we conclude:

Theorem 4.1. Let $h(z)$ be an entire function that satisfies the functional equations

$$
h(z)=-h(z+\pi)=-q^{3 / 2} e^{6 i z} h(z+\pi \tau) .
$$

Then we have the general quintuple product identity

$$
(h(z)+h(-z)) \prod_{n=1}^{\infty}\left(1-q^{n}\right)=2 h(0) \sum_{n=-\infty}^{\infty}(-1)^{n} q^{\left(3 n^{2}+n\right) / 2} \cos (6 n+1) z .
$$

It is easily seen that (4-2) reduces to (4-1) when $h(z)=\theta_{1}(2 z \mid \tau) / \theta_{1}(z \mid \tau)$. Thus (4-2) is really an extension of the quintuple product identity.

Now we will begin to prove Lemma 1.8 using Theorem 4.1. 
Proof. Differentiating this equation twice with respect to $z$ and then setting $z=0$, we have

$$
h^{\prime \prime}(0) \prod_{n=1}^{\infty}\left(1-q^{n}\right)=-h(0) \sum_{n=-\infty}^{\infty}(-1)^{n}(6 n+1)^{2} q^{\left(3 n^{2}+n\right) / 2} .
$$

We recall the Ramanujan identity (from [Ramanujan 1988, page 188] or see also [Berndt and Yee 2003; Liu 2005])

$$
\sum_{n=-\infty}^{\infty}(-1)^{n}(6 n+1)^{2} q^{\left(3 n^{2}+n\right) / 2}=E_{2}(\tau) \prod_{n=1}^{\infty}\left(1-q^{n}\right) .
$$

It follows from these two equations that

$$
h^{\prime \prime}(0)=-h(0) E_{2}(\tau) .
$$

Denote the logarithmic derivative of $h(z)$ by $L(z)$. Then it is easy to show that

$$
h^{\prime \prime}(z)=h(z)\left(L^{2}(z)+L^{\prime}(z)\right) .
$$

Substitute this identity into (4-3) and cancel out the factor $h(0)$ to obtain the interesting identity

$$
L^{2}(0)=-E_{2}(\tau)-L^{\prime}(0) .
$$

Let $h(z)=\theta_{1}(z+x \mid \tau) \theta_{1}(z+y \mid \tau) \theta_{1}(z-x-y \mid \tau)$. Then we find by direct computation that

$$
L(z)=J_{1}(z+x \mid \tau)+J_{1}(z+y \mid \tau)+J_{1}(z-x-y \mid \tau),
$$

where $J_{1}$ is the logarithmic derivative of $\theta_{1}$ with respect to $z$. It follows that

$$
\begin{aligned}
L(0) & =J_{1}(x \mid \tau)+J_{1}(y \mid \tau)-J_{1}(x+y \mid \tau), \\
L^{\prime}(0) & =J_{1}^{\prime}(x \mid \tau)+J_{1}^{\prime}(y \mid \tau)+J_{1}^{\prime}(x+y \mid \tau) .
\end{aligned}
$$

Substituting these two equations into (4-4), we immediately have

$$
\begin{aligned}
\left(J_{1}(x \mid \tau)+J_{1}(y \mid \tau)-\right. & \left.J_{1}(x+y \mid \tau)\right)^{2} \\
& =-E_{2}(\tau)-J_{1}^{\prime}(x \mid \tau)-J_{1}^{\prime}(y \mid \tau)-J_{1}^{\prime}(x+y \mid \tau) .
\end{aligned}
$$

Setting $x=\pi / 7$ and $y=2 \pi / 7$ in this equation, we deduce that

$$
\begin{aligned}
\left(J_{1}\left(\frac{\pi}{7} \mid \tau\right)+J_{1}\left(\frac{2 \pi}{7} \mid \tau\right)\right. & \left.-J_{1}\left(\frac{3 \pi}{7} \mid \tau\right)\right)^{2} \\
& =E_{2}(\tau)-J_{1}^{\prime}\left(\frac{\pi}{7} \mid \tau\right)-J_{1}^{\prime}\left(\frac{2 \pi}{7} \mid \tau\right)-J_{1}^{\prime}\left(\frac{3 \pi}{7} \mid \tau\right) .
\end{aligned}
$$


To simplify this equation, we need some elementary trigonometric sums. With $\omega=\exp (2 \pi i / 7)$, it is well known that for $x \neq 1$,

$$
\prod_{k=1}^{3}\left(1-2 x \cos \frac{2 k \pi}{7}+x^{2}\right)=\frac{1-x^{7}}{1-x} .
$$

Applying the method of partial fraction decomposition, we find that

$$
\sum_{k=1}^{3} \frac{2-2 x \cos (2 k \pi / 7)}{1-2 x \cos (2 k \pi / 7)+x^{2}}=\frac{7}{1-x^{7}}-\frac{1}{1-x} .
$$

Taking the logarithmic derivative of (4-7) and then multiplying the result by $x$, we deduce that

$$
\sum_{k=1}^{3} \frac{2 x^{2}-2 x \cos (2 k \pi / 7)}{1-2 x \cos (2 k \pi / 7)+x^{2}}=-\frac{7 x^{7}}{1-x^{7}}+\frac{x}{1-x} .
$$

If we subtract (4-9) from (4-8) and then divide the result by $2\left(1-x^{2}\right)$, we find

$$
\sum_{k=1}^{3} \frac{1}{1-2 x \cos (2 k \pi / 7)+x^{2}}=\frac{7\left(1+x^{7}\right)}{2\left(1-x^{7}\right)\left(1-x^{2}\right)}-\frac{1}{2(1-x)^{2}} .
$$

Then letting $x \rightarrow 1$ and performing some computations, we find that

$$
\csc ^{2} \frac{\pi}{7}+\csc ^{2} \frac{2 \pi}{7}+\csc ^{2} \frac{3 \pi}{7}=8 .
$$

Equating coefficients of $x$ on both sides of (4-7), we find that

$$
\cos \frac{2 \pi}{7}+\cos \frac{4 \pi}{7}+\cos \frac{6 \pi}{7}=-\frac{1}{2} .
$$

With this value and using the properties of the cosine function, we can easily deduce that

$$
\cos \frac{2 n \pi}{7}+\cos \frac{4 n \pi}{7}+\cos \frac{6 n \pi}{7}=\left\{\begin{array}{rll}
3 & \text { if } n \equiv 0 \quad(\bmod 7), \\
-\frac{1}{2} & \text { if } n \neq 0 \quad(\bmod 7) .
\end{array}\right.
$$

The trigonometric series expansion for the logarithmic derivative of $\theta_{1}$ states [Whittaker and Watson 1996, page 489]

$$
J_{1}(z \mid \tau)=\cot z+4 \sum_{n=1}^{\infty} \frac{q^{n}}{1-q^{n}} \sin 2 n z .
$$

Differentiating this equation with respect to $z$, we conclude that

$$
J_{1}^{\prime}(z \mid \tau)=-\csc ^{2} z+8 \sum_{n=1}^{\infty} \frac{n q^{n}}{1-q^{n}} \cos 2 n z .
$$


With the help of (4-11), (4-13), and (4-15), we deduce that

$$
\begin{aligned}
J_{1}^{\prime}\left(\frac{\pi}{7} \mid \tau\right)+J_{1}^{\prime}\left(\frac{2 \pi}{7} \mid \tau\right)+J_{1}^{\prime}\left(\frac{3 \pi}{7} \mid \tau\right) & \\
& =-8-4 \sum_{n=1}^{\infty} \frac{n q^{n}}{1-q^{n}}+196 \sum_{n=1}^{\infty} \frac{n q^{7 n}}{1-q^{7 n}},
\end{aligned}
$$

and using (3-7) and (4-14), we find that

$$
\begin{aligned}
J_{1}\left(\frac{\pi}{7} \mid \tau\right)+J_{1}\left(\frac{2 \pi}{7} \mid \tau\right) & +J_{1}\left(\frac{3 \pi}{7} \mid \tau\right) \\
= & \cot \frac{\pi}{7}+\cot \frac{2 \pi}{7}-\cot \frac{3 \pi}{7}+2 \sqrt{7} \sum_{n=1}^{\infty}\left(\frac{n}{7}\right) \frac{q^{n}}{1-q^{n}} .
\end{aligned}
$$

Substituting these two equations into (4-6), we immediately find that

$$
\begin{aligned}
\left(\cot \frac{\pi}{7}+\cot \frac{2 \pi}{7}-\cot \frac{3 \pi}{7}+2 \sqrt{7}\right. & \left.\sum_{n=1}^{\infty}\left(\frac{n}{7}\right) \frac{q^{n}}{1-q^{n}}\right)^{2} \\
& =7+28 \sum_{n=1}^{\infty} \frac{n q^{n}}{1-q^{n}}-196 \sum_{n=1}^{\infty} \frac{n q^{7 n}}{1-q^{7 n}} .
\end{aligned}
$$

Putting $q=0$ in this equation, we immediately deduce that

$$
\cot \frac{\pi}{7}+\cot \frac{2 \pi}{7}-\cot \frac{3 \pi}{7}=\sqrt{7}
$$

Substituting this value into (4-18) and canceling out the factor 7 in the resulting equation, we complete the proof of Lemma 1.8.

If (4-19) is substituted into (4-17), we are led to the identity

$$
J_{1}\left(\frac{\pi}{7} \mid \tau\right)+J_{1}\left(\frac{2 \pi}{7} \mid \tau\right)+J_{1}\left(\frac{3 \pi}{7} \mid \tau\right)=\sqrt{7}\left(1+\sum_{n=1}^{\infty}\left(\frac{n}{7}\right) \frac{q^{n}}{1-q^{n}}\right) .
$$

\section{The proof of Lemma 1.9}

Proof. We start by recalling the Guetzlaff identity in Corollary 2.3:

$$
\sqrt{\theta_{2}(0 \mid \tau) \theta_{2}(0 \mid 7 \tau)}+\sqrt{\theta_{4}(0 \mid \tau) \theta_{4}(0 \mid 7 \tau)}=\sqrt{\theta_{3}(0 \mid \tau) \theta_{3}(0 \mid 7 \tau)} .
$$

Divide this equation by $\sqrt{\theta_{3}(0 \mid \tau) \theta_{3}(0 \mid 7 \tau)}$ and use the well-known Jacobi quartic identity, $\theta_{2}^{4}+\theta_{4}^{4}=\theta_{3}^{4}$, to obtain [Berndt 1991, entry 19(i)]

$$
(\alpha \beta)^{1 / 8}+((1-\alpha)(1-\beta))^{1 / 8}=1 .
$$

Combining this identity with the identity $\alpha \beta=t^{8}$ in (1-8), we find that

$$
(1-\alpha)(1-\beta)=(1-t)^{8} \text {. }
$$


Recall from (1-8) that

$$
x_{1}=\left(\frac{\alpha(1-\alpha)}{t(1-t)}\right)^{1 / 3} \text { and } x_{2}=\left(\frac{\beta(1-\beta)}{t(1-t)}\right)^{1 / 3} .
$$

We temporarily define $\bar{t}=t(1-t)$ and multiply $x_{1}$ and $x_{2}$ directly to obtain

$$
x_{1} x_{2}=\bar{t}^{2} .
$$

Using $\alpha \beta=t^{8}$ and (5-2), we find by a direct and elementary calculation that

$$
x_{1}^{3}+x_{2}^{3}=\left(2-7 \bar{t}+4 \bar{t}^{2}\right)^{3}-3 \bar{t}^{2}\left(2-7 \bar{t}+4 \bar{t}^{2}\right) .
$$

On the other hand, we substitute (5-5) in the elementary identity

$$
\left(x_{1}+x_{2}\right)^{3}=x_{1}^{3}+x_{2}^{3}+3 x_{1} x_{2}\left(x_{1}+x_{2}\right)
$$

to obtain

$$
x_{1}^{3}+x_{2}^{3}=\left(x_{1}+x_{2}\right)^{3}-3 \bar{t}^{2}\left(x_{1}+x_{2}\right) .
$$

It follows from these two equations that

$$
\left(x_{1}+x_{2}\right)^{3}-3 \bar{t}^{2}\left(x_{1}+x_{2}\right)=\left(2-7 \bar{t}+4 \bar{t}^{2}\right)^{3}-3 \bar{t}^{2}\left(2-7 \bar{t}+4 \bar{t}^{2}\right) .
$$

Define $\delta(\bar{t})=2-7 \bar{t}+4 \bar{t}^{2}$ and then find from the equation above that

$$
\left(x_{1}+x_{2}-\delta(\bar{t})\right)\left(\left(x_{1}+x_{2}\right)^{2}+\delta(\bar{t})\left(x_{1}+x_{2}\right)+\delta(\bar{t})^{2}-3 \bar{t}^{2}\right)=0 .
$$

Next we will prove

$$
\left(x_{1}+x_{2}\right)^{2}+\delta(\bar{t})\left(x_{1}+x_{2}\right)+\delta(\bar{t})^{2}-3 \bar{t}^{2} \neq 0 .
$$

To this end, we will for the moment assume that $0<q<1$. Then from the Jacobi quartic identity (2-3) we find that $0<\alpha, \beta<1$, and thus we have $0<\bar{t}<1 / 4$. It is easy to see that the discriminant of the quadratic form of $x_{1}+x_{2}$ in (5-9) is

$$
\Delta=-3 \delta^{2}(\bar{t})+12 \bar{t}^{2}=-3\left((2 \bar{t}-5 / 4)^{2}+7 / 16\right)(4 \bar{t}-1)(\bar{t}-2)<0 .
$$

Hence (5-9) holds, and thus from (5-8) we find that

$$
x_{1}+x_{2}=2-7 \bar{t}+4 \bar{t}^{2}=2-7 t+11 t^{2}-8 t^{3}+4 t^{4} .
$$

Combining (5-4) and (5-10), we conclude that $x_{1}$ and $x_{2}$ are the roots of (1-9).

Now we will try to express $x_{1}$ and $x_{2}$ in terms of the parameter $t$. Using the infinite product representations of $\theta_{2}$ and $\theta_{3}$ in Proposition 1.2, we have

$$
\theta_{2}(0 \mid \tau)=\prod_{n=1}^{\infty}\left(1-q^{n}\right)\left(1+q^{n}\right)^{2}
$$




$$
\begin{aligned}
& \theta_{3}(0 \mid \tau)=\prod_{n=1}^{\infty}\left(1-q^{n}\right)\left(1+q^{(n-1 / 2)}\right)^{2}, \\
& \theta_{4}(0 \mid \tau)=\prod_{n=1}^{\infty}\left(1-q^{n}\right)\left(1-q^{(n-1 / 2)}\right)^{2} .
\end{aligned}
$$

It follows that

$$
\alpha=\frac{\theta_{2}^{4}(0 \mid \tau)}{\theta_{3}^{4}(0 \mid \tau)}=16 q^{1 / 2} \prod_{n=1}^{\infty} \frac{\left(1+q^{n}\right)^{8}}{\left(1+q^{n-1 / 2}\right)^{8}} .
$$

Logarithmically differentiating the above with respect to $q$, with the help of the Jacobi four-squares formula in (3-15), we have

$$
2 q \frac{d \alpha}{d q}=\alpha\left(1+8 \sum_{n=1}^{\infty} \frac{2 n q^{n}}{1+q^{n}}-8 \sum_{n=1}^{\infty} \frac{(2 n-1) q^{n-1 / 2}}{1+q^{n-1 / 2}}\right)=\alpha \theta_{4}^{4}(0 \mid \tau) .
$$

So we have $q(d \alpha / d q)>0$ for $0<q<1$, which shows that $\alpha$ is an increasing function of $q$. It is obvious that $0<q^{7}<q$ when $0<q<1$, and hence we have $\beta<\alpha$. Since $\alpha \rightarrow 0$ as $q \rightarrow 0$, we conclude, for very small positive $q$, that $\alpha<1 / 2$. It is easy to see that $\alpha(1-\alpha)$ is increasing when $\alpha<1 / 2$, and so we have $\alpha(1-\alpha)>\beta(1-\beta)$. It follows that $x_{1}>x_{2}$ for very small positive $q$. Solving the quadratic equation (1-9), we arrive at (1-10) and (1-11), proving Lemma 1.9.

Equation (1-9) is the same as [Berndt 1991, Equation 19.6], but our proof is slightly different.

\section{Jacobi's four-squares and the proof of Lemma 1.10}

Proof. From Jacobi's four-squares formula (3-15) it is easy to see that

$$
\begin{aligned}
7 \phi^{4}\left(q^{7}\right)-\phi^{4}(q)=-2\left(1+4 \sum_{n=1}^{\infty} \frac{n q^{n}}{1-q^{n}}-28 \sum_{n=1}^{\infty} \frac{n q^{7 n}}{1-q^{7 n}}\right) \\
+8\left(1+4 \sum_{n=1}^{\infty} \frac{n q^{4 n}}{1-q^{4 n}}-28 \sum_{n=1}^{\infty} \frac{n q^{28 n}}{1-q^{28 n}}\right) .
\end{aligned}
$$

Using Lemmas 1.7 and 1.8 we immediately have

$$
1+4 \sum_{n=1}^{\infty} \frac{n q^{n}}{1-q^{n}}-28 \sum_{n=1}^{\infty} \frac{n q^{7 n}}{1-q^{7 n}}=\left(4 q^{2} \psi\left(q^{2}\right) \psi\left(q^{14}\right)+\phi(q) \phi\left(q^{7}\right)\right)^{2}
$$

Combining (6-1) and (6-2), we find that

$$
\begin{aligned}
7 \phi^{4}\left(q^{7}\right)-\phi^{4}(q)=-2\left(4 q^{2} \psi\left(q^{2}\right)\right. & \left.\psi\left(q^{14}\right)+\phi(q) \phi\left(q^{7}\right)\right)^{2} \\
& +8\left(4 q^{8} \psi\left(q^{8}\right) \psi\left(q^{56}\right)+\phi\left(q^{4}\right) \phi\left(q^{28}\right)\right)^{2}
\end{aligned}
$$


Replacing $q$ by $q^{1 / 2}$ in this identity and then using the relations $\phi\left(q^{1 / 2}\right)=\theta_{3}(0 \mid \tau)$ and $\theta_{2}(0 \mid \tau)=2 q^{1 / 8} \psi(q)$, we have

$$
\begin{aligned}
7 \theta_{3}^{4}(0 \mid 7 \tau)-\theta_{3}^{4}(0 \mid \tau)= & -2\left(\theta_{2}(0 \mid \tau) \theta_{2}(0 \mid 7 \tau)+\theta_{3}(0 \mid \tau) \theta_{3}(0 \mid 7 \tau)\right)^{2} \\
& +8\left(\theta_{2}(0 \mid 4 \tau) \theta_{2}(0 \mid 28 \tau)+\theta_{3}(0 \mid 4 \tau) \theta_{3}(0 \mid 28 \tau)\right)^{2}
\end{aligned}
$$

From the definition of theta functions and by direct computations, we find that

$$
2 \theta_{3}(0 \mid 4 \tau)=\theta_{3}(0 \mid \tau)+\theta_{4}(0 \mid \tau) \text { and } 2 \theta_{2}(0 \mid 4 \tau)=\theta_{3}(0 \mid \tau)-\theta_{4}(0 \mid \tau) .
$$

Using this we find

$$
\begin{aligned}
& 4\left(\theta_{2}(0 \mid 4 \tau) \theta_{2}(0 \mid 28 \tau)+\theta_{3}(0 \mid 4 \tau) \theta_{3}(0 \mid 28 \tau)\right) \\
& =\left(\theta_{3}(0 \mid \tau)-\theta_{4}(0 \mid \tau)\right)\left(\theta_{3}(0 \mid 7 \tau)-\theta_{4}(0 \mid 7 \tau)\right) \\
& +\left(\theta_{3}(0 \mid \tau)+\theta_{4}(0 \mid \tau)\right)\left(\theta_{3}(0 \mid 7 \tau)+\theta_{4}(0 \mid 7 \tau)\right) \\
& =2 \theta_{3}(0 \mid \tau) \theta_{3}(0 \mid 7 \tau)+2 \theta_{4}(0 \mid \tau) \theta_{4}(0 \mid 7 \tau) \text {. }
\end{aligned}
$$

If (6-5) is substituted into (6-4), we get

$$
\begin{aligned}
7 \theta_{3}^{4}(0 \mid 7 \tau)-\theta_{3}^{4}(0 \mid \tau)=- & 2\left(\theta_{2}(0 \mid \tau) \theta_{2}(0 \mid 7 \tau)+\theta_{3}(0 \mid \tau) \theta_{3}(0 \mid 7 \tau)\right)^{2} \\
& +2\left(\theta_{3}(0 \mid \tau) \theta_{3}(0 \mid 7 \tau)+\theta_{4}(0 \mid \tau) \theta_{4}(0 \mid 7 \tau)\right)^{2}
\end{aligned}
$$

Dividing this equation by $\theta_{3}^{2}(0 \mid \tau) \theta_{3}^{2}(0 \mid 7 \tau)$, we deduce that

$$
m-7 / m=-2\left(1+(1-\alpha)^{1 / 4}(1-\beta)^{1 / 4}\right)^{2}+2\left(1+\alpha^{1 / 4} \beta^{1 / 4}\right)^{2} .
$$

Substituting $\alpha \beta=t^{8}$ and $(1-\alpha)(1-\beta)=(1-t)^{8}$ and simplifying gives

$$
m-7 / m=-6+16 t-12 t^{2}+8 t^{3},
$$

which is (1-13). Solving this equation, we arrive at (1-14) and (1-15).

\section{The proof of Theorem 1.11}

We begin this section by proving two theta function identities.

\section{Proposition 7.1.}

$$
\begin{aligned}
& 2 \theta_{2}(x+y \mid 2 \tau) \theta_{2}(x-y \mid 2 \tau)=\theta_{3}(x \mid \tau) \theta_{3}(y \mid \tau)-\theta_{4}(x \mid \tau) \theta_{4}(y \mid \tau), \\
& 2 \theta_{3}(x+y \mid 2 \tau) \theta_{3}(x-y \mid 2 \tau)=\theta_{3}(x \mid \tau) \theta_{3}(y \mid \tau)+\theta_{4}(x \mid \tau) \theta_{4}(y \mid \tau) .
\end{aligned}
$$

Proof. In [Liu 2007] we proved the general theta function identity

$$
\begin{aligned}
\left(h_{1}(x \mid \tau)-\right. & \left.h_{1}(-x \mid \tau)\right)\left(h_{2}(y \mid \tau)-h_{2}(-y \mid \tau)\right) \\
-\left(h_{2}(x \mid \tau)-\right. & \left.h_{2}(-x \mid \tau)\right)\left(h_{1}(y \mid \tau)-h_{1}(-y \mid \tau)\right) \\
& =C \theta_{1}(x \mid \tau) \theta_{1}(y \mid \tau) \theta_{1}(x+y \mid \tau) \theta_{1}(x-y \mid \tau),
\end{aligned}
$$


where $C$ is independent of $x$ and $y$, and $h_{1}$ and $h_{2}$ are two entire functions of $z$ satisfying the functional equations

$$
h_{k}(z \mid \tau)=-h_{k}(z+\pi \mid \tau)=-q^{3 / 2} e^{6 i z} h_{k}(z+\pi \tau \mid \tau) \quad \text { for } k=1,2 .
$$

Take $2 h_{1}(z)=\theta_{1}(z \mid \tau) \theta_{4}(z \mid \tau / 2)$ and $2 h_{2}(z)=\theta_{1}(z \mid \tau) \theta_{3}(z \mid \tau / 2)$. Then (7-1) gives

$$
\theta_{3}(y \mid \tau / 2) \theta_{4}(x \mid \tau / 2)-\theta_{3}(x \mid \tau / 2) \theta_{4}(y \mid \tau / 2)=C \theta_{1}(x+y \mid \tau) \theta_{1}(x-y \mid \tau) .
$$

If we set $y=\pi \tau / 4$ and $x=0$, then the second term of the left side vanishes. Thus we have

$$
q^{-1 /(16)} \theta_{2}(0 \mid \tau / 2) \theta_{4}(0 \mid \tau / 2)=-C \theta_{1}^{2}(\pi \tau / 4 \mid \tau) .
$$

With the infinite product representations of theta functions in Proposition 1.2, we find that

$$
2 \theta_{1}^{2}(\pi \tau / 4 \mid \tau)=-q^{-1 /(16)} \theta_{2}(0 \mid \tau / 2) \theta_{4}(0 \mid \tau / 2)=-2(q ; q)_{\infty}^{2}\left(q^{1 / 4} ; q^{1 / 2}\right)^{2} .
$$

Thus we have $C=2$. Substituting this value in (7-2), we find that [Liu 2009]

$$
\theta_{3}(y \mid \tau / 2) \theta_{4}(x \mid \tau / 2)-\theta_{3}(x \mid \tau / 2) \theta_{4}(y \mid \tau / 2)=2 \theta_{1}(x+y \mid \tau) \theta_{1}(x-y \mid \tau) .
$$

If we replace $\tau$ by $2 \tau$ and replace $x$ by $x+\pi / 2$ and $x+(\pi+2 \pi \tau) / 2$, then the resulting equations are just the two identities in Proposition 7.1, respectively.

Proof of Theorem 1.11. We will start with the identity in Lemma 1.7, which can be written as

$$
1+2 \sum_{n=1}^{\infty}\left(\frac{n}{7}\right) \frac{q^{n}}{1-q^{n}}=\theta_{3}(0 \mid 2 \tau) \theta_{3}(0 \mid 14 \tau)+\theta_{2}(0 \mid 2 \tau) \theta_{2}(0 \mid 14 \tau) .
$$

Putting $x=y=0$ in Proposition 7.1, we immediately deduce that

$$
2 \theta_{3}^{2}(0 \mid 2 \tau)=\theta_{3}^{2}(0 \mid \tau)+\theta_{4}^{2}(0 \mid \tau) \quad \text { and } \quad 2 \theta_{2}^{2}(0 \mid 2 \tau)=\theta_{3}^{2}(0 \mid \tau)-\theta_{4}^{2}(0 \mid \tau) .
$$

Using these two equations and the definition of $\alpha$ in (1-8), we have

$$
\begin{aligned}
& \theta_{3}(0 \mid 2 \tau)=\theta_{3}(0 \mid \tau) \sqrt{\frac{1+\sqrt{1-\alpha}}{2}}, \\
& \theta_{2}(0 \mid 2 \tau)=\theta_{3}(0 \mid \tau) \sqrt{\frac{1-\sqrt{1-\alpha}}{2}} .
\end{aligned}
$$


Replacing $\tau$ by $7 \tau$ in these two formulas and using the definition of $\beta$ in (1-8), we find that

$$
\begin{aligned}
& \theta_{3}(0 \mid 14 \tau)=\theta_{3}(0 \mid 7 \tau) \sqrt{\frac{1+\sqrt{1-\beta}}{2}}, \\
& \theta_{2}(0 \mid 14 \tau)=\theta_{3}(0 \mid 7 \tau) \sqrt{\frac{1-\sqrt{1-\beta}}{2}} .
\end{aligned}
$$

Substituting (7-4) and (7-5) into the right side of (7-3), we find that

$$
\frac{1}{2} \theta_{3}(0 \mid \tau) \theta_{3}(0 \mid 7 \tau)(A+B)
$$

where

$$
A:=\sqrt{(1+\sqrt{1-\alpha})(1+\sqrt{1-\beta})} \quad \text { and } \quad B:=\sqrt{(1-\sqrt{1-\alpha})(1-\sqrt{1-\beta})} .
$$

By direct computations, we find that

$$
A B=\sqrt{\alpha \beta}=t^{4} \quad \text { and } \quad A^{2}+B^{2}=2+2 \sqrt{(1-\alpha)(1-\beta)}=2+2(1-t)^{4} .
$$

It follows that $A+B=2\left(1-t+t^{2}\right)$. Substituting this into (7-6), we deduce the identity [Berndt 1991, entry 5(ii)]

$$
1+2 \sum_{n=1}^{\infty}\left(\frac{n}{7}\right) \frac{q^{n}}{1-q^{n}}=\theta_{3}(0 \mid \tau) \theta_{3}(0 \mid 7 \tau)\left(1-t+t^{2}\right) .
$$

Comparing (1-10) with (1-15), and (1-11) with (1-14), we find that

$$
m=\frac{1-4 x_{2}}{1-2 t} \text { and } \quad \frac{7}{m}=-\frac{1-4 x_{1}}{1-2 t} .
$$

We add these two identities together and immediately have

$$
m+\frac{7}{m}=\frac{4\left(x_{1}-x_{2}\right)}{1-2 t} \text {. }
$$

Multiplying the first identity in (7-8) by $4 x_{1}$ and the second by $4 x_{2}$ and then adding the two resulting identities together gives

$$
4 m x_{1}+\frac{28 x_{2}}{m}=\frac{4\left(x_{1}-x_{2}\right)}{1-2 t} .
$$

Comparing last two equations, we find that $m+7 / m=4 m x_{1}+28 x_{2} / m$. We square both sides of this and then subtract $16 x_{1} x_{2}$ from the result to find

$$
16 m^{2} x^{2}+208 x_{1} x_{2}+784 \frac{x_{2}^{2}}{m^{2}}=(m-7 / m)^{2}-16 x_{1} x_{2}+28 .
$$


Substituting

$$
m-7 / m=-6+16 t-12 t^{2}+8 t^{3} \quad \text { and } \quad x_{1} x_{2}=t^{2}\left(1-t^{2}\right)
$$

into the right side of (7-9) and simplifying gives the value $64\left(1-t+t^{2}\right)^{3}$. Thus

$$
\frac{1}{4}\left(m^{2} x_{1}^{2}+13 x_{1} x_{2}+49 x_{2}^{2} / m^{2}\right)=\left(1-t+t^{2}\right)^{3} .
$$

We multiply this through by $\theta_{3}^{3}(0 \mid \tau) \theta_{3}^{3}(0 \mid 7 \tau)$ and then combine the result with (7-7) to obtain

$$
\left(1+2 \sum_{n=1}^{\infty}\left(\frac{n}{7}\right) \frac{q^{n}}{1-q^{n}}\right)^{3}=\frac{1}{4} \theta_{3}^{3}(0 \mid \tau) \theta_{3}^{3}(0 \mid 7 \tau)\left(m^{2} x_{1}^{2}+13 x_{1} x_{2}+49 \frac{x_{2}^{2}}{m^{2}}\right)
$$

Using the definitions of $x_{1}, x_{2}, t$, and $m$, and the infinite product representations of $\theta_{2}, \theta_{3}$, and $\theta_{4}$, we find that

$$
\begin{aligned}
\theta_{3}^{3}(0 \mid \tau) \theta_{3}^{3}(0 \mid 7 \tau) m^{2} x_{1}^{2} & =\frac{\theta_{3}^{7}(0 \mid \tau)}{\theta_{3}(0 \mid 7 \tau)}\left(\frac{\alpha^{7}(1-\alpha)^{7}}{\beta(1-\beta)}\right)^{1 / 12} \\
& =\left(\frac{\theta_{2}^{28}(0 \mid \tau) \theta_{3}^{28}(0 \mid \tau) \theta_{4}^{28}(0 \mid \tau)}{\theta_{2}^{4}(0 \mid 7 \tau) \theta_{3}^{4}(0 \mid 7 \tau) \theta_{4}^{4}(0 \mid 7 \tau)}\right)^{1 / 12} \\
& =4 \prod_{n=1}^{\infty} \frac{(1-q)^{7}}{\left(1-q^{7 n}\right)}=4 \frac{\eta^{7}(\tau)}{\eta(7 \tau)}
\end{aligned}
$$

In the same way we can find that

$$
\begin{aligned}
\theta_{3}^{3}(0 \mid \tau) \theta_{3}^{3}(0 \mid 7 \tau) x_{1} x_{2} & =4 \eta^{3}(\tau) \eta^{3}(7 \tau), \\
\theta_{3}^{3}(0 \mid \tau) \theta_{3}^{3}(0 \mid 7 \tau) x_{2}^{2} / m^{2} & =4 \eta^{7}(7 \tau) / \eta(\tau) .
\end{aligned}
$$

Substituting (7-12) and (7-13) into (7-11) completes the proof of Theorem 1.11.

\section{The Ramanujan-Watson modular equation}

In this section we prove the Ramanujan-Watson modular equation in Theorem 1.12 using Corollary 1.4 and Theorem 1.11.

Proof. For brevity we will define $u, v$, and $w$ as

$$
\begin{aligned}
u & :=u(\tau)=\theta_{1}\left(\frac{\pi}{7} \mid \tau\right), \\
v & :=v(\tau)=\theta_{1}\left(\frac{2 \pi}{7} \mid \tau\right), \\
w & :=w(\tau)=\theta_{1}\left(\frac{3 \pi}{7} \mid \tau\right) .
\end{aligned}
$$


Choosing $h(z)=\theta_{1}^{3}(z \mid \tau)$ in Corollary 1.4 , setting $x=\pi / 7$ and $y=2 \pi / 7$, and simplifying, we immediately have

$$
u^{3} v-v^{3} w+w^{3} u=0
$$

which gives a parametrization of the Klein quartic curve $X^{3} Y+Y^{3} Z+Z^{3} X=0$; see for example [Elkies 1999; Lachaud 2005]. It is easy to show, using the infinite product representation of $\theta_{1}$ in Proposition 1.2, that

$$
u v w=\sqrt{7} \eta^{2}(\tau) \eta(7 \tau)
$$

We further set

$$
a:=a(\tau)=v / u, \quad b:=b(\tau)=-w / v, \quad c:=c(\tau)=u / w .
$$

If we multiply directly these formulas together, we immediately deduce that

$$
a b c=-1 .
$$

With the help of (8-3), it is easily seen that (8-2) can be rewritten as

$$
a b^{2}-a^{2}+c=0 .
$$

Multiplying this by $a^{-1}$ and then using (8-4) in the resulting equation, we find that

$$
b c^{2}-b^{2}+a=0 .
$$

In the same way we multiply (8-5) by $c$ and use (8-4) to obtain

$$
c a^{2}-c^{2}+b=0 \text {. }
$$

Using the quintuple product identity (4-1), we can easily show that [Liu 2003]

$$
a+b+c=1+\rho
$$

where $\rho$ is defined as

$$
\rho(\tau)=7 \eta(49 \tau) / \eta(\tau) .
$$

We multiply (8-5) by $a,(8-6)$ by $b$, and (8-7) by $c$, and add the resulting equations together to obtain

$$
\left(a^{2} b^{2}+b^{2} c^{2}+c^{2} a^{2}\right)-\left(a^{3}+b^{3}+c^{3}\right)+a b+b c+c a=0 .
$$

Define

$$
P:=a+b+c=1+\rho, \quad Q:=a b+b c+c a, \quad R:=a b c=-1 .
$$


Using the theory of elementary symmetric polynomials and using (8-4)-(8-8), we find that

$$
\begin{aligned}
a^{2} b^{2}+b^{2} c^{2}+c^{2} a^{2} & =Q^{2}+2(1+\rho), \\
a^{3}+b^{3}+c^{3} & =(1+\rho)^{3}-3 Q(1+\rho)-3 .
\end{aligned}
$$

If these are combined with (8-10), we conclude that

$$
Q^{2}+(3 \rho+4) Q-\left(\rho^{3}+3 \rho^{2}+\rho-4\right)=0 .
$$

We solve this equation for $Q$, and, with some analysis, find this:

Proposition 8.1. $Q=-\frac{1}{2}(3 \rho+4)-\frac{1}{2} \sqrt{4 \rho^{3}+21 \rho+28 \rho}$.

Set

$$
y_{1}:=y_{1}(\tau)=a^{3} b, \quad y_{2}:=y_{2}(\tau)=b^{3} c, \quad y_{3}:=y_{3}(\tau)=c^{3} a .
$$

Using (8-4)-(8-7) and some straightforward evaluations, we deduce that

$$
y_{1} y_{2}=-y_{1}-1, \quad y_{2} y_{3}=-y_{2}-1, \quad y_{3} y_{1}=-y_{3}-1, \quad y_{1} y_{2} y_{3}=1 .
$$

It is easily shown by direct computation that for any complex numbers $X, Y$, and $Z$,

$$
\begin{aligned}
& (X+Y+Z)^{3} \\
& =X^{3}+Y^{3}+Z^{3}+6 X Y Z+3 X^{2} Y+3 X^{2} Z+3 Y^{2} X+3 Y^{2} Z+3 Z^{2} X+3 Z^{2} Y .
\end{aligned}
$$

Choosing $X=\sqrt[3]{y_{1}^{2} y_{2}}, Y=\sqrt[3]{y_{2}^{2} y_{3}}$, and $Z=\sqrt[3]{y_{3}^{2} y_{1}}$, and simplifying with the help of (8-15), we find that

$$
\left(\sqrt[3]{y_{1}^{2} y_{2}}+\sqrt[3]{y_{2}^{2} y_{3}}+\sqrt[3]{y_{3}^{2} y_{1}}\right)^{3}=-\left(y_{1}+y_{2}+y_{3}\right)^{2}-3\left(y_{1}+y_{2}+y_{3}\right)-9 .
$$

Employing the definitions of $u, v, w, a, b$, and $c$, by direct computations, we have

$$
\begin{aligned}
& \sqrt[3]{y_{1}^{2} y_{2}}=-7^{1 / 6} \eta^{2 / 3}(\tau) \eta^{1 / 3}(7 \tau) \frac{w}{u^{2}}, \\
& \sqrt[3]{y_{2}^{2} y_{3}}=7^{1 / 6} \eta^{2 / 3}(\tau) \eta^{1 / 3}(7 \tau) \frac{u}{v^{2}}, \\
& \sqrt[3]{y_{3}^{2} y_{1}}=-7^{1 / 6} \eta^{2 / 3}(\tau) \eta^{1 / 3}(7 \tau) \frac{v}{w^{2}} .
\end{aligned}
$$

If these three formulas are substituted into (8-15), we find that

(8-16) $\left(y_{1}+y_{2}+y_{3}\right)^{2}+3\left(y_{1}+y_{2}+y_{3}\right)+9=\sqrt{7} \eta^{2}(\tau) \eta(7 \tau)\left(\frac{w}{u^{2}}-\frac{u}{v^{2}}+\frac{v}{w^{2}}\right)^{3}$.

We recall the following theta function identity, which can be easily derived from Corollary 1.6; see [Liu 2005; McCullough and Shen 1994]. 


\section{Proposition 8.2.}

$$
\begin{aligned}
J_{1}(x \mid \tau)+J_{1}(y \mid \tau)+J_{1}(z \mid \tau)- & J_{1}(x+y+z \mid \tau) \\
& =\frac{\theta_{1}^{\prime}(0 \mid \tau) \theta_{1}(x+y \mid \tau) \theta_{1}(x+z \mid \tau) \theta_{1}(y+z \mid \tau)}{\theta_{1}(x \mid \tau) \theta_{1}(y \mid \tau) \theta_{1}(z \mid \tau) \theta_{1}(x+y+z \mid \tau)} .
\end{aligned}
$$

We set $(x, y, z)$ equal to

$$
(\pi / 7,-3 \pi / 7,-3 \pi / 7), \quad(\pi / 7,-2 \pi / 7,-2 \pi / 7), \quad(\pi / 7, \pi / 7,-2 \pi / 7),
$$

in the above equation, and then adding the resulting equations together, we find

$$
2 J_{1}\left(\frac{\pi}{7} \mid \tau\right)+2 J_{1}\left(\frac{2 \pi}{7} \mid \tau\right)-2 J_{1}\left(\frac{3 \pi}{7} \mid \tau\right)=\theta_{1}^{\prime}(0 \mid \tau)\left(\frac{w}{u^{2}}-\frac{u}{v^{2}}+\frac{v}{w^{2}}\right) .
$$

Combining this with (4-20), we find that

$$
1+2 \sum_{n=1}^{\infty}\left(\frac{n}{7}\right) \frac{q^{n}}{1-q^{n}}=\frac{\eta^{3}(\tau)}{\sqrt{7}}\left(\frac{w}{u^{2}}-\frac{u}{v^{2}}+\frac{v}{w^{2}}\right) .
$$

If this is combined with Theorem 1.11, we find the following identity, which is the same as [Liu 2003, (1.27)]:

$$
\left(\frac{w}{u^{2}}-\frac{u}{v^{2}}+\frac{v}{w^{2}}\right)^{3}=7 \sqrt{7} \eta^{-9}(\tau) \lambda^{-1}\left(\mu^{2}+13 \mu+49\right) .
$$

Combining this with (8-16), we conclude that

$$
\left(y_{1}+y_{2}+y_{3}\right)^{2}+3\left(y_{1}+y_{2}+y_{3}\right)+9=49\left(1+13 \mu^{-1}+49 \mu^{-2}\right) .
$$

Solving this equation, we deduce the next proposition [Liu 2003, (5.14)].

Proposition 8.3. $y_{1}+y_{2}+y_{3}=-8-49 \mu^{-1}$.

Now we are ready to derive the Ramanujan-Watson modular equation.

Adding (8-5), (8-6), and (8-7) together, and using (8-11), we find that

$$
a b^{2}+b c^{2}+c a^{2}=P^{2}-2 Q-P .
$$

We multiply $P=a+b+c$ and $Q=a b+b c+c a$ directly and, with the help of $a b c=-1$, we obtain

$$
P Q=a c^{2}+c a^{2}+b a^{2}+a b^{2}+b c^{2}+c a^{2}-3 .
$$

Substituting (8-20) into this, we find that

$$
a c^{2}+c a^{2}+b a^{2}=-P^{2}+P Q+P+Q+3 .
$$

Multiplying (8-5) by $b,(8-6)$ by $c$, and (8-7) by $a$, and adding the three resulting identities, we obtain

$$
a b^{3}+b c^{3}+c a^{3}=a c^{2}+c b^{2}+b a^{2}-a b-b c-c a .
$$


Substituting (8-21) and $a b+b c+c a=Q$ into the above, we conclude that

$$
a b^{3}+b c^{3}+c a^{3}=-P^{2}+P Q+P+Q+3 .
$$

With the help of $a b c=-1$, by a direct computation, we find that

$$
a^{3} b+b^{3} c+c^{3} a=(a+b+c)(a b+b c+c a)-a b^{3}-b c^{3}-c a^{3}+a+b+c .
$$

Substituting $P=a+b+c, Q=a b+b c+c a$, and (8-22) into the above, we find that

$$
a^{3} b+b^{3} c+c^{3} a=P^{2} Q+P^{2}-2 Q^{2}-P Q-Q-3 .
$$

Using $y_{1}=a^{3} b, y_{2}=b^{3} c$, and $y_{3}=c^{3} a$ in the equation above, we conclude that

$$
y_{1}+y_{2}+y_{3}=P^{2} Q+P^{2}-2 Q^{2}-P Q-Q-3 .
$$

Combining this with Proposition 8.3, we have

$$
P^{2} Q+P^{2}-2 Q^{2}-P Q-Q-3=-8-49 \mu^{-1} .
$$

Substituting $P=1+\rho$ and the value of $Q$ in Proposition 8.1 in the right hand side of the equation above, we have

$$
-\frac{1}{2}\left(\rho^{2}+7 \rho+7\right) \sqrt{4 \rho^{2}+21 \rho+28 \rho}-\frac{1}{2}\left(7 \rho^{3}+35 \rho^{2}+49 \rho\right)-8 .
$$

We obtain, from these two equations, the following result.

Proposition 8.4. $\left(\rho^{2}+7 \rho+7\right) \sqrt{4 \rho^{2}+21 \rho+28 \rho}+\left(7 \rho^{3}+35 \rho^{2}+49 \rho\right)=98 \mu^{-1}$.

The well-known modular transformation for the Dedekind $\eta$-function is given by

$$
\eta\left(-\frac{1}{\tau}\right)=\sqrt{-i \tau} \eta(\tau)
$$

It follows that

$$
\rho\left(-\frac{1}{7 \tau}\right)=\nu(\tau) \quad \text { and } \quad \mu\left(-\frac{1}{7 \tau}\right)=49 \mu^{-1}(\tau) .
$$

Replacing $\tau$ by $-1 / 7 \tau$ in Proposition 8.4 and then applying the above transformation formulas, we complete the proof of Theorem 1.12.

\section{Theta function identities related to modular equations of degree 7}

Let $a, b$, and $c$ be defined as in (8-3) and let $F(a, b, c)$ be a symmetric function in the variables $a, b$, and c. Then from (8-4), (8-8), and Proposition 8.1, we know that $F(a, b, c)$ can be expressed in terms of $\rho$. In particular, $K_{n}:=K_{n}(\tau)=a^{n}+b^{n}+c^{n}$ can be written in terms of $\rho$. 
Theorem 9.1. Let $a, b, c$ be defined as in (8-3). Then we have the recurrence relation

$$
K_{n+3}=(1+\rho) K_{n+2}+\frac{1}{2}\left(3 \rho+4+\sqrt{4 \rho^{3}+21 \rho+28 \rho}\right) K_{n+1}-K_{n},
$$

with the boundary conditions

$$
K_{-1}=\frac{1}{2}(3 \rho+4)+\frac{1}{2} \sqrt{4 \rho^{3}+21 \rho+28 \rho}, \quad K_{0}=3, \quad K_{1}=1+\rho .
$$

Proof. From (8-4), (8-8), and Proposition 8.1, we find that $a, b$, and $c$ are the three roots of the cubic equation

$$
x^{3}-(1+\rho) x^{2}-\frac{1}{2}\left(3 \rho+4+\sqrt{4 \rho^{3}+21 \rho+28 \rho}\right) x+1=0 .
$$

Multiplying both sides of the equation above by $x^{n}$ gives

$$
x^{3+n}-(1+\rho) x^{2+n}-\frac{1}{2}\left(3 \rho+4+\sqrt{4 \rho^{3}+21 \rho+28 \rho}\right) x^{n+1}+x^{n}=0 .
$$

Replacing $x$ by each of $a, b, c$ in the equation above and then adding the three resulting equations, we obtain (9-1), completing the proof of Theorem 9.1.

Theorem 9.1 allows us to derive many theta function identities. In particular,

$$
\begin{aligned}
& K_{2}=\rho^{2}+5 \rho+5+\sqrt{4 \rho^{3}+21 \rho+28 \rho}, \\
& K_{3}=\rho^{3}+\frac{15}{2} \rho^{2}+\frac{27}{2} \rho+4+\frac{3}{2}(1+\rho) \sqrt{4 \rho^{3}+21 \rho+28 \rho} .
\end{aligned}
$$

From the definitions of $a, b$, and $c$ in (8-3), it is easily seen that

$$
K_{n}(i \infty)=\left(\frac{\sin 2 \pi / 7}{\sin \pi / 7}\right)^{n}+(-1)^{n}\left(\frac{\sin 3 \pi / 7}{\sin 2 \pi / 7}\right)^{n}+\left(\frac{\sin \pi / 7}{\sin 3 \pi / 7}\right)^{n} .
$$

When $q=0$, (9-1) will reduce to the following recurrence relation.

Corollary 9.2. With $K_{n}(i \infty)$ defined as in (9-2), we have

$$
K_{n+3}(i \infty)=K_{n+2}(i \infty)+2 K_{n+1}(i \infty)-K_{n}(i \infty),
$$

with the initial values $K_{-1}(i \infty)=2, K_{0}(i \infty)=3, K_{1}(i \infty)=1$.

From this recurrence we can readily find the following finite trigonometric sums:

$$
\begin{aligned}
& K_{2}(i \infty)=\left(\frac{\sin 2 \pi / 7}{\sin \pi / 7}\right)^{2}+\left(\frac{\sin 3 \pi / 7}{\sin 2 \pi / 7}\right)^{2}+\left(\frac{\sin \pi / 7}{\sin 3 \pi / 7}\right)^{2}=5, \\
& K_{3}(i \infty)=\left(\frac{\sin 2 \pi / 7}{\sin \pi / 7}\right)^{3}-\left(\frac{\sin 3 \pi / 7}{\sin 2 \pi / 7}\right)^{3}+\left(\frac{\sin \pi / 7}{\sin 3 \pi / 7}\right)^{3}=4, \\
& K_{4}(i \infty)=\left(\frac{\sin 2 \pi / 7}{\sin \pi / 7}\right)^{4}+\left(\frac{\sin 3 \pi / 7}{\sin 2 \pi / 7}\right)^{4}+\left(\frac{\sin \pi / 7}{\sin 3 \pi / 7}\right)^{4}=13,
\end{aligned}
$$




$$
\begin{aligned}
& K_{5}(i \infty)=\left(\frac{\sin 2 \pi / 7}{\sin \pi / 7}\right)^{5}-\left(\frac{\sin 3 \pi / 7}{\sin 2 \pi / 7}\right)^{5}+\left(\frac{\sin \pi / 7}{\sin 3 \pi / 7}\right)^{5}=16, \\
& K_{6}(i \infty)=\left(\frac{\sin 2 \pi / 7}{\sin \pi / 7}\right)^{6}+\left(\frac{\sin 3 \pi / 7}{\sin 2 \pi / 7}\right)^{6}+\left(\frac{\sin \pi / 7}{\sin 3 \pi / 7}\right)^{6}=38 \\
& K_{7}(i \infty)=\left(\frac{\sin 2 \pi / 7}{\sin \pi / 7}\right)^{7}-\left(\frac{\sin 3 \pi / 7}{\sin 2 \pi / 7}\right)^{7}+\left(\frac{\sin \pi / 7}{\sin 3 \pi / 7}\right)^{7}=57 .
\end{aligned}
$$

We add the first three formulas in (8-13) together and then use Proposition 8.3 to find that

$$
y_{1} y_{2}+y_{2} y_{3}+y_{3} y_{1}=-\left(y_{1}+y_{2}+y_{3}\right)-3=5+49 \mu^{-1} \text {. }
$$

If $F\left(y_{1}, y_{2}, y_{3}\right)$ is a symmetric function of $y_{1}, y_{2}$, and $y_{3}$, then from the theory of symmetric functions and (9-4), and $y_{1} y_{2} y_{3}=1$, we know that $F\left(y_{1}, y_{2}, y_{3}\right)$ can be written in terms of $\mu^{-1}$. So we can find infinitely many theta function identities involving $y_{1}, y_{2}$, and $y_{3}$. Define $S_{n}$ as

$$
S_{n}:=S_{n}(\tau)=y_{1}^{n}+y_{2}^{n}+y_{3}^{n} \text {. }
$$

Theorem 9.3. Let $y_{1}, y_{2}$, and $y_{3}$ be defined as in (8-12), and let $S_{n}$ be as in (9-5). Then we have

$$
S_{n+3}=-\left(8+49 \mu^{-1}\right) S_{n+2}-\left(5+49 \mu^{-1}\right) S_{n+1}+S_{n},
$$

with the initial values,

$$
S_{0}=3, \quad S_{1}=-8-49 \mu^{-1}, \quad S_{2}=54+2 \times 7^{3} \mu^{-1}+7^{4} \mu^{-2} .
$$

Proof. Using (9-4) and $y_{1} y_{2} y_{3}=1$, we know that $y_{1}, y_{2}$, and $y_{3}$ are the roots of the cubic equation

$$
x^{3}+\left(8+49 \mu^{-1}\right) x^{2}+\left(5+49 \mu^{-1}\right) x-1=0,
$$

which gives $x^{n+3}+\left(8+49 \mu^{-1}\right) x^{n+2}+\left(5+49 \mu^{-1}\right) x^{n+1}-x^{n}=0$. Replacing $x$ with $y_{1}, y_{2}$, and then $y_{3}$, and adding the resulting equations, we arrive at (9-6). This completes the proof of Theorem 9.3.

It is easy to see from the definition of $y_{1}, y_{2}$, and $y_{3}$ in (8-12) that

$$
\begin{aligned}
& y_{1}=-\sqrt{7} \eta^{2}(\tau) \eta(7 \tau) \frac{\theta_{1}(2 \pi / 7 \mid \tau)}{\theta_{1}^{4}(\pi / 7 \mid \tau)}, \\
& y_{2}=-\sqrt{7} \eta^{2}(\tau) \eta(7 \tau) \frac{\theta_{1}(3 \pi / 7 \mid \tau)}{\theta_{1}^{4}(2 \pi / 7 \mid \tau)}, \\
& y_{3}=\sqrt{7} \eta^{2}(\tau) \eta(7 \tau) \frac{\theta_{1}(\pi / 7 \mid \tau)}{\theta_{1}^{4}(3 \pi / 7 \mid \tau)} .
\end{aligned}
$$


Letting $q=0$ and with the help of the infinite product representation of $\theta_{1}$, we immediately have

$$
\begin{aligned}
& y_{1}(i \infty)=-\frac{\sqrt{7}}{8} \frac{\sin (2 \pi / 7)}{\sin ^{4}(\pi / 7)}, \\
& y_{2}(i \infty)=-\frac{\sqrt{7}}{8} \frac{\sin (3 \pi / 7)}{\sin ^{4}(2 \pi / 7)}, \\
& y_{3}(i \infty)=\frac{\sqrt{7}}{8} \frac{\sin (\pi / 7)}{\sin ^{4}(3 \pi / 7)} .
\end{aligned}
$$

It follows that $S_{n}(i \infty)=(-\sqrt{7} / 8)^{n} T_{n}$, where

$$
T_{n}=\frac{\sin ^{n}(2 \pi / 7)}{\sin ^{4 n}(\pi / 7)}+(-1)^{n} \frac{\sin ^{n}(\pi / 7)}{\sin ^{4 n}(3 \pi / 7)}+\frac{\sin ^{n}(3 \pi / 7)}{\sin ^{4 n}(2 \pi / 7)} .
$$

Thus putting $q=0$ in (9-6) and simplifying, we get the recurrence relation for $T_{n}$.

Corollary 9.4. Let $T_{n}$ be defined as in (9-8). Then we have

$$
T_{n+3}=\frac{64}{\sqrt{7}} T_{n+2}-\frac{320}{7} T_{n+1}-\frac{512}{7 \sqrt{7}} T_{n},
$$

with the initial values $T_{-1}=-\frac{5}{8} \sqrt{7}, T_{0}=3$ and $T_{1}=\frac{64}{\sqrt{7}}$.

We may use this recurrence relation to compute infinitely many trigonometric sums. For example, we have

$$
\begin{aligned}
& T_{2}=\frac{\sin ^{2}(2 \pi / 7)}{\sin ^{8}(\pi / 7)}+\frac{\sin ^{2}(\pi / 7)}{\sin ^{8}(3 \pi / 7)}+\frac{\sin ^{2}(3 \pi / 7)}{\sin ^{8}(2 \pi / 7)}=\frac{3456}{7} \\
& T_{3}=\frac{\sin ^{3}(2 \pi / 7)}{\sin ^{12}(\pi / 7)}-\frac{\sin ^{3}(\pi / 7)}{\sin ^{12}(3 \pi / 7)}+\frac{\sin ^{3}(3 \pi / 7)}{\sin ^{12}(2 \pi / 7)}=\frac{199168}{7 \sqrt{7}} .
\end{aligned}
$$

For a systematic study of this type of trigonometric sums, see [Beck et al. 2005; Berndt and Zaharescu 2004; Chan 2007].

Now we will begin proving the following identity of Ramanujan [1988, page 53]; see also [Raghavan 1986; Liu 2003].

Proposition 9.5. $8-7 \sum_{n=1}^{\infty}\left(\frac{n}{7}\right) \frac{n^{2} q^{n}}{1-q^{n}}=\lambda^{-1}\left(8 \mu^{2}+49 \mu\right)$.

Proof. The addition formula for the Weierstrass $\sigma$-function can be written in terms of $\theta_{1}$ as (see for example [Liu 2005, (4.10)])

$$
J_{1}^{\prime}(x \mid \tau)-J_{1}^{\prime}(y \mid \tau)=4 \eta^{6}(\tau) \frac{\theta_{1}(x+y \mid \tau) \theta_{1}(x-y \mid \tau)}{\theta_{1}^{2}(x \mid \tau) \theta_{1}^{2}(y \mid \tau)} .
$$


Dividing both sides by $x-y$ and then letting $y \rightarrow x$ yields

$$
J_{1}^{\prime \prime}(x \mid \tau)=8 \eta^{9}(\tau) \frac{\theta_{1}(2 x \mid \tau)}{\theta_{1}^{4}(x \mid \tau)} .
$$

Taking $x=\pi / 7,2 \pi / 7$, and then $-3 \pi / 7$, in this equation and adding the three resulting equations, we have

$$
8-7 \sum_{n=1}^{\infty}\left(\frac{n}{7}\right) \frac{n^{2} q^{n}}{1-q^{n}}=\sqrt{7} \eta^{9}(\tau)\left(\frac{\theta_{1}(2 \pi / 7 \mid \tau)}{\theta_{1}^{4}(\pi / 7 \mid \tau)}-\frac{\theta_{1}(\pi / 7 \mid \tau)}{\theta_{1}^{4}(3 \pi / 7 \mid \tau)}+\frac{\theta_{1}(3 \pi / 7 \mid \tau)}{\theta_{1}^{4}(2 \pi / 7 \mid \tau)}\right)
$$

Substituting (9-7) into the right side, we find that

$$
8-7 \sum_{n=1}^{\infty}\left(\frac{n}{7}\right) \frac{n^{2} q^{n}}{1-q^{n}}=-\frac{\eta^{7}(\tau)}{\eta(7 \tau)}\left(y_{1}+y_{2}+y_{3}\right) .
$$

Combining this with Proposition 8.3, we arrive at the identity of Ramanujan.

Theorem 9.6. Let $C_{n}=K_{7 n}=a^{7 n}+b^{7 n}+c^{7 n}$. Then we have

$$
C_{n+3}=C_{1} C_{n+2}+C_{-1} C_{n+1}-C_{n},
$$

with the initial value $C_{0}=3$, and $C_{1}$ and $C_{-1}$ being given by

$$
\begin{aligned}
C_{1} & =57+2 \times 7^{3} \mu^{-1}+7^{4} \mu^{-2}, \\
C_{-1} & =289+18 \times 7^{3} \mu^{-1}+19 \times 7^{4} \mu^{-2}+7^{6} \mu^{-3} .
\end{aligned}
$$

Proof. Multiplying (8-5) with $a b$, (8-6) with $b c$, and (8-7) with $a c$, and using the definitions of $y_{1}, y_{2}, y_{3}$ in (8-12), we find that

$$
a^{2} b^{3}=y_{1}+1, \quad b^{2} c^{3}=y_{2}+1, \quad c^{2} a^{3}=y_{3}+1 .
$$

In the same way, we multiply (8-5) with $b^{3},(8-6)$ with $c^{3}$, and (8-7) with $a^{3}$, to obtain

$$
a b^{5}=a^{2} b^{3}-y_{2}, \quad b c^{5}=b^{2} c^{3}-y_{3}, \quad c a^{5}=c^{2} a^{3}-y_{1} .
$$

Combining these two sets of equations, we deduce that

$$
a b^{5}=y_{1}-y_{2}+1, \quad b c^{5}=y_{2}-y_{3}+1, \quad c a^{5}=y_{3}-y_{1}+1 .
$$

Multiplying (8-5) by $a^{5}$, (8-6) by $b^{3}$, and (8-7) by $c^{3}$, and using the definitions of $y_{1}, y_{2}, y_{3}$, we obtain

$$
a^{7}=a^{5} c+y_{1}^{2}, \quad b^{7}=b^{5} a+y_{2}^{2}, \quad c^{7}=c^{5} b=y_{3}^{2} .
$$

Combining the two sets of equations above, we find that (see also [Liu 2003])

(9-13) $a^{7}=y_{1}^{2}-y_{1}+y_{3}+1, \quad b^{7}=y_{2}^{2}-y_{2}+y_{1}+1, \quad c^{7}=y_{3}^{2}-y_{3}+y_{2}+1$. 
Add these three equations above together and use (9-4) to obtain

$$
C_{1}=a^{7}+b^{7}+c^{7}=y_{1}^{2}+y_{2}^{2}+y_{3}^{2}+3=57+2 \times 7^{3} \mu^{-1}+7^{4} \mu^{-2} .
$$

Using (8-13) and (9-13), by direct computations we find that

$$
a^{7} b^{7}=y_{1}\left(y_{1}+1\right)^{2}, \quad b^{7} c^{7}=y_{2}\left(y_{2}+1\right)^{2}, \quad c^{7} a^{7}=y_{3}\left(y_{3}+1\right)^{2} .
$$

Adding these three equations together and using (9-4), we find that

$$
(a b)^{7}+(b c)^{7}+(c a)^{7}=-289-18 \times 7^{3} \mu^{-1}-19 \times 7^{4} \mu^{-2}-7^{6} \mu^{-3} .
$$

Combining $(a b c)^{7}=-1$ with the equation above, we find that

$$
C_{-1}=289+18 \times 7^{3} \mu^{-1}+19 \times 7^{4} \mu^{-2}+7^{6} \mu^{-3} .
$$

Then using (9-14), (9-15), and $(a b c)^{7}=-1$, we find that $a^{7}, b^{7}$, and $c^{7}$ are the three roots of the cubic equation

$$
X^{3}-C_{1} X^{2}-C_{-1} X+1=0 .
$$

Using the same argument that we used to prove Theorems 9.1 and 9.3, we can complete the proof of Theorem 9.6.

This recurrence relation will enable us to derive infinitely many theta function identities. For example, we have

$$
\begin{aligned}
C_{3}=234609+24306 & \times 7^{3} \mu^{-1}+52671 \times 7^{4} \mu^{-2} \\
& +8879 \times 7^{6} \mu^{-3}+858 \times 7^{8} \mu^{-4}+45 \times 7^{10} \mu^{-5}+7^{12} \mu^{-6} .
\end{aligned}
$$

Putting $q=0$, we immediately have

$$
C_{n}(i \infty)=\left(\frac{\sin 2 \pi / 7}{\sin \pi / 7}\right)^{7 n}+(-1)^{n}\left(\frac{\sin 3 \pi / 7}{\sin 2 \pi / 7}\right)^{7 n}+\left(\frac{\sin \pi / 7}{\sin 3 \pi / 7}\right)^{7 n} .
$$

Letting $q=0$ in (9-12), we immediately deduce the next corollary.

Corollary 9.7. Let $C_{n}(i \infty)$ be defined as in (9-18). Then we have

$$
C_{n+3}(i \infty)=57 C_{n+2}(i \infty)+289 C_{n+1}(i \infty)-C_{n}(i \infty),
$$

with the initial values $C_{-1}(i \infty)=289, C_{0}(i \infty)=3$ and $C_{1}(i \infty)=57$.

This can be used to derive infinitely many finite trigonometric sum formulas. For example,

$$
\begin{aligned}
& C_{2}(i \infty)=\left(\frac{\sin 2 \pi / 7}{\sin \pi / 7}\right)^{14}+\left(\frac{\sin 3 \pi / 7}{\sin 2 \pi / 7}\right)^{14}+\left(\frac{\sin \pi / 7}{\sin 3 \pi / 7}\right)^{14}=3827 \\
& C_{3}(i \infty)=\left(\frac{\sin 2 \pi / 7}{\sin \pi / 7}\right)^{21}-\left(\frac{\sin 3 \pi / 7}{\sin 2 \pi / 7}\right)^{21}+\left(\frac{\sin \pi / 7}{\sin 3 \pi / 7}\right)^{21}=234609 .
\end{aligned}
$$




\section{Ramanujan's identities related to modular equations of degree 7}

In this section, we explore how our theta function identities behave when acted on by imaginary transformations. We need the well-known imaginary transformation formula for the Jacobi theta function $\theta_{1}$ [Whittaker and Watson 1996, page 475]:

$$
\theta_{1}\left(\frac{z}{\tau} \mid-\frac{1}{\tau}\right)=-i \sqrt{-i \tau} \exp \left(\frac{i z^{2}}{\pi \tau}\right) \theta_{1}(z \mid \tau) .
$$

Replacing $\tau$ with $7 \tau$ in the above equation and then taking $z=r \pi \tau$ in the resulting equation, we find that

$$
\theta_{1}\left(\frac{r \pi}{7} \mid-\frac{1}{7 \tau}\right)=-i \sqrt{-7 i \tau} q^{\left(r^{2} / 14\right)} \theta_{1}(r \pi \tau \mid 7 \tau) .
$$

In contrast to $a, b$, and $c$ defined in (8-3), we now introduce $a, \mathfrak{b}$, and $\mathbb{C}$ defined by

(10-3) $\mathrm{a}=q^{\frac{3}{14}} \frac{\theta_{1}(2 \pi \tau \mid 7 \tau)}{\theta_{1}(\pi \tau \mid 7 \tau)}, \quad \mathbb{b}=-q^{\frac{5}{14}} \frac{\theta_{1}(3 \pi \tau \mid 7 \tau)}{\theta_{1}(2 \pi \tau \mid 7 \tau)}, \quad \mathbb{C}=q^{-\frac{8}{14}} \frac{\theta_{1}(\pi \tau \mid 7 \tau)}{\theta_{1}(3 \pi \tau \mid 7 \tau)}$.

For convenience, in what follows we will sometimes adopt the compact notation $[z ; q]_{\infty}$ defined as

$$
[z ; q]_{\infty}=\prod_{n=0}^{\infty}\left(1-z q^{n}\right)\left(1-q^{n} / z\right) \text { for } z \neq 0 .
$$

Using the infinite product representation for $\theta_{1}$ in Proposition 1.2, we can find the infinite product representations for $\mathfrak{a}, \mathfrak{b}$, and $\mathbb{C}$ :

$$
\mathrm{a}=q^{-2 / 7} \frac{\left[q^{2} ; q^{7}\right]_{\infty}}{\left[q ; q^{7}\right]_{\infty}}, \quad \mathbb{b}=-q^{-1 / 7} \frac{\left[q^{3} ; q^{7}\right]_{\infty}}{\left[q^{2} ; q^{7}\right]_{\infty}}, \quad \mathbb{C}=q^{3 / 7} \frac{\left[q ; q^{7}\right]_{\infty}}{\left[q^{3} ; q^{7}\right]_{\infty}}
$$

With the help of (10-2), it is easy to establish the next proposition.

Proposition 10.1. With $a, b$, and $c$ defined as in (8-3) and $\mathrm{a}$, $\mathfrak{b}$, and $\mathbb{C}$ as in (10-3), we have

$$
a(-1 / 7 \tau)=\mathrm{a}, \quad b(-1 / 7 \tau)=\mathfrak{b}, \quad c(-1 / 7 \tau)=\mathbb{C} .
$$

Theorem 10.2. Let $\mathrm{a}, \mathfrak{b}$, and $\mathbb{C}$ be defined as in (10-3). Then we have Ramanujan's identity [Berndt 1991, (18.1)]

$$
\mathrm{a}+\mathfrak{b}+\mathbb{C}=1+v .
$$

Proof. Replacing $\tau$ by $-1 /(7 \tau)$ in (8-8) and then using the modular transformation for the $\eta$-function and the modular transformations in Proposition 10.1, we immediately obtain Theorem 10.2. 
Theorem 10.3. Let $\mathrm{a}, \mathfrak{b}$, and $\mathbb{C}$ be defined as in (10-3). Then we have Ramanujan's identities [Berndt 1991, (18. 2) and (18.3)]

$$
\begin{aligned}
a^{7}+b^{7}+\mathbb{C}^{7} & =57+14 \mu+\mu^{2}, \\
a^{7} b^{7}+b^{7} \mathbb{C}^{7}+\mathbb{c}^{7} a^{7} & =-289-126 \mu-19 \mu^{2}-\mu^{3} .
\end{aligned}
$$

Proof. Using the modular transformation for the $\eta$-function, we easily find that

$$
\mu\left(-\frac{1}{7 \tau}\right)=49 \mu^{-1}(\tau) .
$$

Replacing $\tau$ by $-1 /(7 \tau)$ in (9-14) and (9-15), using the modular transformation for the $\eta$-function, and the modular transformations in Proposition 10.1 and (10-5), we complete the proof of Theorem 10.3.

Multiplying the three identities in (10-4) together, we easily find that

$$
\mathrm{a} \mathfrak{b} \mathbb{C}=-1 .
$$

Set

$$
\mathbb{C}_{n}=\mathbb{C}_{n}(\tau)=\mathrm{a}^{7 n}+\mathfrak{b}^{7 n}+\mathbb{C}^{7 n} .
$$

Then from Theorem 10.3 and (10-6), we find that the values of $\mathbb{C}_{-1}$ and $\mathbb{C}_{1}$ are

$$
\begin{aligned}
& \mathbb{C}_{-1}=289+126 \mu+19 \mu^{2}+\mu^{3}, \\
& \mathbb{C}_{1}=57+14 \mu+\mu^{2} .
\end{aligned}
$$

Theorem 10.4. Let $\mathbb{C}_{n}$ be defined as in (10-7). Then we have the recurrence relation

$$
\mathbb{C}_{n+3}=\mathbb{C}_{1} \mathbb{C}_{n+2}+\mathbb{C}_{-1} \mathbb{C}_{n+1}-\mathbb{C}_{n},
$$

with the initial value $\mathbb{C}_{0}=3$, and $\mathbb{C}_{-1}$ and $\mathbb{C}_{1}$ being given in (10-8).

Proof. From Theorem 10.3 and (10-6), we find that $\mathrm{a}^{7}, \mathrm{~b}^{7}$, and $\mathbb{C}^{7}$ are the three roots of the cubic equation

$$
X^{3}-\mathbb{C}_{1} X^{2}-\mathbb{C}_{-1} X+1=0 .
$$

From this equation we can easily find the recurrence relation in Theorem 10.4.

Theorem 10.4 allows us to derive infinitely many theta function identities. For example, we have

$(10-9) \quad \mathbb{C}_{3}:=234609+170142 \mu+52671 \mu^{2}+8879 \mu^{3}+858 \mu^{4}+45 \mu^{5}+\mu^{6}$.

Proposition 10.5. Let $y_{1}, y_{2}$, and $y_{3}$ be defined as in (8-12), and let $\mathbb{y}_{1}, \mathbb{y}_{2}$, and $\mathbb{y}_{3}$ be defined as

$$
\mathrm{y}_{1}=\mathrm{a}^{3} \mathfrak{b}, \quad \mathrm{y}_{2}=\mathfrak{b}^{3} \mathbb{C}, \quad \mathrm{y}_{3}=\mathbb{C}^{3} \mathrm{a}
$$


Then we have the modular transformation formulas

$$
\mathbb{y}_{1}=y_{1}(-1 / 7 \tau), \quad \mathbb{y}_{2}=y_{2}(-1 / 7 \tau), \quad \mathbb{y}_{3}=y_{3}(-1 / 7 \tau) \text {. }
$$

With their help, we can find the infinite product representations for $\mathbb{y}_{1}, \mathbb{y}_{2}, \mathbb{y}_{3}$ :

$$
\begin{aligned}
& \mathrm{y}_{1}=-q^{-3 / 4} \frac{\eta(\tau)}{\eta(7 \tau)} \frac{\left[q^{2} ; q^{7}\right]_{\infty}}{\left[q ; q^{7}\right]_{\infty}^{4}}, \\
& \mathrm{y}_{2}=-q^{1 / 4} \frac{\eta(\tau)}{\eta(7 \tau)} \frac{\left[q^{3} ; q^{7}\right]_{\infty}}{\left[q^{2} ; q^{7}\right]_{\infty}^{4}}, \\
& \mathbb{y}_{3}=q^{5 / 4} \frac{\eta(\tau)}{\eta(7 \tau)} \frac{\left[q ; q^{7}\right]_{\infty}}{\left[q^{3} ; q^{7}\right]_{\infty}^{4}} .
\end{aligned}
$$

Theorem 10.6. Let $\mathrm{y}_{1}, \mathbb{y}_{2}$, and $\mathrm{y}_{3}$ be defined as in (10-10). Then we have the identities [Berndt and Zhang 1994, (4.22) and (4.23); Garvan 1984, page 323]

$$
\begin{aligned}
\mathbb{y}_{1}+\mathbb{y}_{2}+\mathbb{y}_{3} & =-\mu-8, \\
\mathbb{y}_{1} \mathbb{y}_{2}+\mathbb{y}_{2} \mathbb{y}_{3}+\mathbb{y}_{3} \mathbb{y}_{1} & =\mu+5 .
\end{aligned}
$$

Proof. Replacing $\tau$ by $-1 /(7 \tau)$ in Proposition 8.3 and then using the transformations in Proposition 10.5 and (10-5), we immediately obtain the first identity. In the same way, by replacing $\tau$ by $-1 /(7 \tau)$ in (9-4), we obtain the second identity.

Set $\mathbb{S}_{n}:=\mathbb{S}_{n}(\tau)=\mathbb{y}_{1}^{n}+\mathbb{y}_{2}^{n}+\mathbb{y}_{3}^{n}$. Then using Theorem 10.6 and $\mathbb{y}_{1} \mathbb{y}_{2} \mathbb{y}_{3}=1$, we can establish the next theorem.

Theorem 10.7. We have the recurrence relation

$$
\mathbb{S}_{n+3}=-(\mu+8) \mathbb{S}_{n+2}-(\mu+5) \mathbb{S}_{n+1}+\mathbb{S}_{n},
$$

with the initial values $\mathbb{S}_{0}=3, \mathbb{S}_{1}=-\mu-8, \mathbb{S}_{2}=\mu^{2}+14 \mu+54$.

From this recurrence relation we can derive infinitely many theta function identities. For example,

$$
\begin{aligned}
& \mathbb{S}_{3}=-\mu^{3}-21 \mu^{2}-153 \mu-389, \\
& \mathbb{S}_{4}=\mu^{4}+28 \mu^{3}+302 \mu^{2}+1488 \mu+2834, \\
& \mathbb{S}_{5}=-\mu^{5}-35 \mu^{4}-500 \mu^{3}-3645 \mu^{2}-13570 \mu-20673 .
\end{aligned}
$$

Theorem 10.8. Let $\lambda$ and $\mu$ be defined as in (1-3). Then we have

$$
\begin{aligned}
q^{-13 / 4}\left[q ; q^{7}\right]_{\infty}^{-7}-q^{11 / 4}\left[q^{2} ; q^{7}\right]_{\infty}^{-7}-q^{11 / 2} & {\left[q^{3} ; q^{7}\right]_{\infty}^{-7} } \\
& =\mu^{-7 / 12}\left(\mu^{2}+13 \mu+49\right)^{1 / 3}(\mu+7) .
\end{aligned}
$$


Proof. Choosing $X=\sqrt[3]{\mathrm{y}_{1}^{5} \mathrm{y}_{2}}, Y=\sqrt[3]{\mathrm{y}_{2}^{5} \mathrm{y}_{3}}$, and $Z=\sqrt[3]{\mathrm{y}_{3}^{5} \mathrm{y}_{1}}$ in (8-13), and making use of $\mathbb{y}_{1} \mathbb{y}_{2}=-\mathbb{y}_{\mathbb{1}}-1, \mathbb{y}_{2} \mathbb{Y}_{3}=-\mathbb{y}_{2}-1, \quad \mathbb{y}_{3} \mathbb{y}_{1}=-\mathbb{y}_{3}-1$, and $\mathbb{y}_{1} \mathbb{y}_{2} \mathbb{y}_{3}=1$, we deduce that

$$
\left(\sqrt[3]{\mathrm{y}_{1}^{5} \mathbb{Y}_{2}}+\sqrt[3]{y_{2}^{5} \mathbb{y}_{3}}+\sqrt[3]{\mathbb{y}_{3}^{5} \mathbb{y}_{1}}\right)^{3}=-\mathbb{S}_{5}-\mathbb{S}_{4}+3 \mathbb{S}_{3}+3 \mathbb{S}_{2}+3 \mathbb{S}_{1}-3 .
$$

Substituting the values of $\mathbb{S}_{r}$ for $r=1,2,3,4,5$ in Theorem 10.7, and (10-12) into the equation above, we find that

$$
\left(\sqrt[3]{\mathrm{y}_{1}^{5} \mathrm{y}_{2}}+\sqrt[3]{\mathrm{y}_{2}^{5} \mathbb{Y}_{3}}+\sqrt[3]{\mathrm{y}_{3}^{5} \mathbb{y}_{1}}\right)^{3}=\left(\mu^{2}+13 \mu+49\right)(\mu+7)^{3} .
$$

Substituting (10-11) into the left hand side of the equation above and simplifying, we complete the proof of Theorem 10.8.

\section{Logarithmic differentiation and two elliptic theta function identities}

We will begin this section by proving Theorem 1.15 with the help of the general quintuple product identity and the method of logarithmic differentiation.

Proof. We recall the general quintuple product identity given in Theorem 4.1:

$(11-1) \quad(h(z)+h(-z)) \prod_{n=1}^{\infty}\left(1-q^{n}\right)=2 h(0) \sum_{n=-\infty}^{\infty}(-1)^{n} q^{\left(3 n^{2}+n\right) / 2} \cos (6 n+1) z$.

Differentiating this equation four times with respect to $z$ and then setting $z=0$, we find that

$$
h^{(4)}(0)=h(0) \prod_{n=1}^{\infty}\left(1-q^{n}\right)^{-1} \sum_{n=-\infty}^{\infty}(-1)^{n}(6 n+1)^{4} q^{\left(3 n^{2}+n\right) / 2} .
$$

Let $E_{2 k}$ be the normalized Eisenstein series defined in Definition 1.14. Then we have Ramanujan's identity (see for example, [Berndt and Yee 2003; Liu 2005])

$$
\prod_{n=1}^{\infty}\left(1-q^{n}\right)^{-1} \sum_{n=-\infty}^{\infty}(-1)^{n}(6 n+1)^{4} q^{\left(3 n^{2}+n\right) / 2}=3 E_{2}^{2}(\tau)-2 E_{4}(\tau) .
$$

If this is substituted into (11-2), we deduce that

$$
h^{(4)}(0)=h(0)\left(3 E_{2}^{2}(\tau)-2 E_{4}(\tau)\right) .
$$

Let $L(z)$ be the logarithmic derivative of $h(z)$. Then by a direct calculation, we find that

$$
h^{(4)}(z)=h(z) \times\left(L^{4}(z)+6 L^{2}(z) L^{\prime}(z)+4 L(z) L^{\prime \prime}(z)+3 L^{\prime}(z)^{2}+L^{\prime \prime \prime}(z)\right) .
$$

Choosing $h(z)=\theta_{1}(z+x \mid \tau) \theta_{1}(z+y \mid \tau) \theta_{1}(z-x-y \mid \tau)$, we find that

$$
L(z)=J_{1}(z+x \mid \tau)+J_{1}(z+y \mid \tau)+J_{1}(z-x-y \mid \tau) .
$$


It follows that

$$
\begin{aligned}
L(0) & =J_{1}(x \mid \tau)+J_{1}(y \mid \tau)-J_{1}(x+y \mid \tau), \\
L^{\prime}(0) & =J_{1}^{\prime}(x \mid \tau)+J_{1}^{\prime}(y \mid \tau)+J_{1}^{\prime}(x+y \mid \tau), \\
L^{\prime \prime}(0) & =J_{1}^{\prime \prime}(x \mid \tau)+J_{1}^{\prime \prime}(y \mid \tau)-J_{1}^{\prime}(x+y \mid \tau), \\
L^{\prime \prime \prime}(0) & =J_{1}^{\prime \prime \prime}(x \mid \tau)+J_{1}^{\prime \prime \prime}(y \mid \tau)+J_{1}^{\prime \prime \prime}(x+y \mid \tau) .
\end{aligned}
$$

Putting $z=0$ in (11-4), substituting the equations above in the resulting equation, and finally comparing with (11-3), we find that

$$
\begin{aligned}
3 E_{2}^{2}(\tau)-2 E_{4}(\tau)= & \left(J_{1}(x \mid \tau)+J_{1}(y \mid \tau)-J_{1}(x+y \mid \tau)\right)^{4} \\
& +3\left(J_{1}^{\prime}(x \mid \tau)+J_{1}^{\prime}(y \mid \tau)+J_{1}^{\prime}(x+y \mid \tau)\right)^{2} \\
& +J_{1}^{\prime \prime \prime}(x \mid \tau)+J_{1}^{\prime \prime \prime}(y \mid \tau)+J_{1}^{\prime \prime \prime}(x+y \mid \tau) \\
& +6\left(J_{1}(x \mid \tau)+J_{1}(y \mid \tau)-J_{1}(x+y \mid \tau)\right)^{2} \\
& \times\left(J_{1}^{\prime}(x \mid \tau)+J_{1}^{\prime}(y \mid \tau)+J_{1}^{\prime}(x+y \mid \tau)\right) \\
& +4\left(J_{1}(x \mid \tau)+J_{1}(y \mid \tau)-J_{1}(x+y \mid \tau)\right) \\
& \times\left(J_{1}^{\prime \prime}(x \mid \tau)+J_{1}^{\prime \prime}(y \mid \tau)-J_{1}^{\prime \prime}(x+y \mid \tau)\right) .
\end{aligned}
$$

To derive Theorem 1.15 we also need the identity in (4-5), which states $\left(J_{1}(x \mid \tau)+J_{1}(y \mid \tau)-J_{1}(x+y \mid \tau)\right)^{2}=-E_{2}(\tau)-J_{1}^{\prime}(x \mid \tau)-J_{1}^{\prime}(y \mid \tau)-J_{1}^{\prime}(x+y \mid \tau)$.

Eliminating $J_{1}^{\prime}(x \mid \tau)+J_{1}^{\prime}(y \mid \tau)+J_{1}^{\prime}(x+y \mid \tau)$ from the last two equations, we immediately arrive at Theorem 1.15 .

The proof of Theorem 1.16 is similar to the proof of Theorem 1.15 and so we only sketch it.

Proof. Differentiating both sides of (11-1) six times with respect to $z$ and then setting $z=0$, we find that

$$
h^{(6)}(0)=-h(0) \prod_{n=1}^{\infty}\left(1-q^{n}\right)^{-1} \sum_{n=-\infty}^{\infty}(-1)^{n}(6 n+1)^{6} q^{\left(3 n^{2}+n\right) / 2} .
$$

The substitution of Ramanujan's identity (see for example [Berndt and Yee 2003; Liu 2005])

$$
\begin{array}{r}
\prod_{n=1}^{\infty}\left(1-q^{n}\right)^{-1} \sum_{n=-\infty}^{\infty}(-1)^{n}(6 n+1)^{6} q^{\left(3 n^{2}+n\right) / 2} \\
=15 E_{2}^{3}(\tau)-30 E_{2}(\tau) E_{4}(\tau)+16 E_{6}(\tau)
\end{array}
$$

into (11-5) gives

$$
h^{(6)}(0)=-h(0)\left(15 E_{2}^{3}(\tau)-30 E_{2}(\tau) E_{4}(\tau)+16 E_{6}(\tau)\right) .
$$


Choosing $h(z)=\theta_{1}(z+x \mid \tau) \theta_{1}(z+y \mid \tau) \theta_{1}(z-x-y \mid \tau)$ and then using the same argument that we used to derive Theorem 1.15, we can derive Theorem 1.16. Thus we complete the proof of Theorem 1.16.

\section{Eisenstein series identities related to the modular equation of degree 7}

We begin with the Laurent expansion of the logarithmic derivative of $\theta_{1}$ [Liu 2005, page 8; Liu 2007, page 400].

Proposition 12.1. Let $B_{k}$ be the Bernoulli numbers defined as in Definition 1.13, and let $E_{2 k}$ be the Eisenstein series defined as in Definition 1.14. Then we have

$$
\begin{aligned}
J_{1}(z \mid \tau) & =\frac{1}{z}-\frac{1}{3} E_{2}(\tau) z-\frac{1}{45} E_{4}(\tau) z^{3}-\frac{2}{945} E_{6}(\tau) z^{5}+\cdots \\
& =\frac{1}{z}+\sum_{k=1}^{\infty}(-1)^{k} \frac{2^{2 k} B_{2 k}}{(2 k) !} E_{2 k}(\tau) z^{2 k-1} .
\end{aligned}
$$

Using the infinite product representations of theta functions, we deduce the next proposition.

Proposition 12.2 [Enneper 1890, pages 246-250].

$$
\begin{gathered}
\prod_{r=1}^{3} \theta_{1}\left(z+\frac{r \pi}{7} \mid \tau\right) \theta_{1}\left(z-\frac{r \pi}{7} \mid \tau\right)=-\prod_{n=1}^{\infty} \frac{\left(1-q^{n}\right)^{7}}{\left(1-q^{7 n}\right)} \frac{\theta_{1}(7 z \mid 7 \tau)}{\theta_{1}(z \mid \tau)}, \\
\prod_{r=1}^{3} \theta_{1}(z-r \pi \tau \mid 7 \tau) \theta_{1}(z+r \pi \tau \mid 7 \tau)=\prod_{n=1}^{\infty} \frac{\left(1-q^{7 n}\right)^{7}}{\left(1-q^{n}\right)} \frac{\theta_{1}(z \mid \tau)}{\theta_{1}(z \mid 7 \tau)} .
\end{gathered}
$$

Logarithmically differentiating these equations with respect to $z$, we deduce the next proposition.

$$
\text { Proposition 12.3. } \begin{aligned}
& \sum_{r=1}^{3}\left(J_{r}\left(z+\frac{r \pi}{7} \mid \tau\right)+J_{r}\left(z-\frac{r \pi}{7} \mid \tau\right)\right)=7 J_{1}(7 z \mid \tau)-J_{1}(z \mid \tau), \\
& \sum_{r=1}^{3}\left(J_{r}(z+r \pi \tau \mid 7 \tau)+J_{r}(z-r \pi \tau \mid 7 \tau)\right)=J_{1}(z \mid \tau)-J_{1}(z \mid 7 \tau) .
\end{aligned}
$$

Substituting the Laurent series in Proposition 12.1 into the right hand sides of the two equations in Proposition 12.3 and equating like powers of $z$, we deduce the next proposition.

$$
\text { Proposition 12.4. } \begin{aligned}
& \sum_{r=1}^{3} J_{r}^{(2 k-1)}\left(\frac{r \pi}{7} \mid \tau\right)=\frac{(-4)^{k-1}}{k} B_{2 k}\left(E_{2 k}(\tau)-7^{2 k} E_{2 k}(7 \tau)\right), \\
& \sum_{r=1}^{3} J_{r}^{(2 k-1)}(r \pi \tau \mid 7 \tau)=\frac{(-4)^{k-1}}{k} B_{2 k}\left(E_{2 k}(7 \tau)-E_{2 k}(\tau)\right) .
\end{aligned}
$$


In particular,

$$
\begin{aligned}
& \sum_{r=1}^{3} J_{r}^{\prime}\left(\frac{r \pi}{7} \mid \tau\right)=\frac{1}{6}\left(E_{2}(\tau)-7^{2} E_{2}(7 \tau)\right), \\
& \sum_{r=1}^{3} J_{r}^{\prime \prime \prime}\left(\frac{r \pi}{7} \mid \tau\right)=\frac{1}{15}\left(E_{4}(\tau)-7^{4} E_{4}(7 \tau)\right) \\
& \sum_{r=1}^{3} J_{r}^{(5)}\left(\frac{r \pi}{7} \mid \tau\right)=\frac{8}{63}\left(E_{6}(\tau)-7^{6} E_{6}(7 \tau)\right), \\
& \sum_{r=1}^{3} J_{r}^{\prime}(r \pi \tau \mid 7 \tau)=\frac{1}{6}\left(E_{2}(7 \tau)-E_{2}(\tau)\right) \\
& \sum_{r=1}^{3} J_{r}^{\prime \prime \prime}(r \pi \tau \mid 7 \tau)=\frac{1}{15}\left(E_{4}(7 \tau)-E_{4}(\tau)\right) \\
& \sum_{r=1}^{3} J_{r}^{(5)}(r \pi \tau \mid 7 \tau)=\frac{8}{63}\left(E_{6}(7 \tau)-E_{6}(\tau)\right)
\end{aligned}
$$

Using some trigonometric identities and some elementary and direct calculations, we find that

$$
\begin{aligned}
& \sum_{r=1}^{3}\left(\frac{r}{7}\right) J_{1}\left(\frac{r \pi}{7} \mid \tau\right)=\sqrt{7}\left(1+2 \sum_{n=1}^{\infty}\left(\frac{n}{7}\right) \frac{q^{n}}{1-q^{n}}\right), \\
& \sum_{r=1}^{3}\left(\frac{r}{7}\right) J_{1}^{\prime \prime}\left(\frac{r \pi}{7} \mid \tau\right)=\frac{8}{\sqrt{7}}\left(8-7 \sum_{n=1}^{\infty}\left(\frac{n}{7}\right) \frac{n^{2} q^{n}}{1-q^{n}}\right), \\
& \sum_{r=1}^{3}\left(\frac{r}{7}\right) J_{1}^{(4)}\left(\frac{r \pi}{7} \mid \tau\right)=32 \sqrt{7}\left(16+\sum_{n=1}^{\infty}\left(\frac{n}{7}\right) \frac{n^{4} q^{n}}{1-q^{n}}\right), \\
& \sum_{r=1}^{3}\left(\frac{r}{7}\right) J_{1}(r \pi \tau \mid 7 \tau)=-i\left(1+2 \sum_{n=1}^{\infty}\left(\frac{n}{7}\right) \frac{q^{n}}{1-q^{n}}\right), \\
& \sum_{r=1}^{3}\left(\frac{r}{7}\right) J_{1}^{\prime \prime}(r \pi \tau \mid 7 \tau)=8 i \sum_{r=1}^{6}\left(\frac{r}{7}\right) \sum_{n=1}^{\infty} \frac{n^{2} q^{r n}}{1-q^{7 n}}, \\
& \sum_{r=1}^{3}\left(\frac{r}{7}\right) J_{1}^{(4)}(r \pi \tau \mid 7 \tau)=-32 i \sum_{r=1}^{\infty}\left(\frac{r}{7}\right) \sum_{n=1}^{\infty} \frac{n^{4} q^{r n}}{1-q^{7 n}} .
\end{aligned}
$$

Motivated by Ramanujan's work and [Liu 2003], Chan and Cooper [2008] gave a systematic study of this type of series, and succeeded in expressing the series $584-\sum_{n=1}^{\infty}\left(\frac{n}{7}\right) n^{6} q^{n} /\left(1-q^{n}\right)$ in terms of the Dedekind $\eta$-function. 
Now we will use Theorem 1.15 to prove the first two identities in Theorem 1.17. Proof. Putting $x=\pi / 7, y=2 \pi / 7$ in Theorem 1.15 , we immediately have

$$
\begin{aligned}
2 E_{4}(\tau)+\sum_{r=1}^{3} J_{r}^{\prime \prime \prime}\left(\frac{r \pi}{7} \mid \tau\right)=2\left(\sum_{r=1}^{3}\left(\frac{r}{7}\right) J_{1}\left(\frac{r \pi}{7} \mid \tau\right)\right)^{4} & \\
& -4\left(\sum_{r=1}^{3}\left(\frac{r}{7}\right) J_{1}\left(\frac{r \pi}{7} \mid \tau\right)\right)\left(\sum_{r=1}^{3}\left(\frac{r}{7}\right) J_{1}^{\prime \prime}\left(\frac{r \pi}{7} \mid \tau\right)\right) .
\end{aligned}
$$

Substituting the second identity in (12-1) and the first two identities in (12-2) into this equation, we find that

$$
\begin{aligned}
\frac{1}{15}\left(31 E_{4}(\tau)-7^{4} E_{4}(7 \tau)\right)= & 98\left(1+2 \sum_{n=1}^{\infty}\left(\frac{n}{7}\right) \frac{q^{n}}{1-q^{n}}\right)^{4} \\
& -32\left(1+2 \sum_{n=1}^{\infty}\left(\frac{n}{7}\right) \frac{q^{n}}{1-q^{n}}\right)\left(8-7 \sum_{n=1}^{\infty}\left(\frac{n}{7}\right) \frac{n^{2} q^{n}}{1-q^{n}}\right) .
\end{aligned}
$$

Using Theorem 1.11 and Proposition 9.5 in the right side of this equation, we get

$$
\begin{aligned}
& \frac{1}{15}\left(7^{4} E_{4}(7 \tau)-31 E_{4}(\tau)\right) \\
& =2 \lambda^{-4 / 3}\left(\mu^{2}+13 \mu+49\right)^{1 / 3}\left(79 \mu^{2}+147 \mu-2401\right) .
\end{aligned}
$$

Replacing $\tau$ by $-1 /(7 \tau)$ in this equation and using the modular transformations

$$
\begin{aligned}
& E_{4}(-1 / \tau)=\tau^{4} E_{4}(\tau), \quad E_{4}(-1 / 7 \tau)=7^{4} \tau^{4} E_{4}(\tau), \\
& \mu(-1 / 7 \tau)=49 \mu^{-1}(\tau), \quad \lambda(-1 / 7 \tau)=-\sqrt{7} i \tau^{-3} \eta(7 \tau) / \eta^{7}(\tau),
\end{aligned}
$$

in the resulting equation, and simplifying, we deduce that

(12-5) $\frac{1}{15}\left(E_{4}(\tau)-31 E_{4}(7 \tau)\right)=2 \lambda^{-4 / 3}\left(\mu^{2}+13 \mu+49\right)^{1 / 3}\left(-\mu^{2}+3 \mu+79\right)$.

Solving the linear system of equations (12-3) and (12-5) for $E_{4}(\tau)$ and $E_{4}(7 \tau)$ will give the first two identities in Theorem 1.17.

Proposition 12.5. Let $\left(\frac{r}{7}\right)$ be the Legendre symbol modulo 7. Then we have

$$
\sum_{r=1}^{3}\left(\frac{r}{7}\right) J_{1}^{(2 k)}\left(\frac{r \pi}{7} \mid-\frac{1}{7 \tau}\right)=(7 \tau)^{2 k+1} \sum_{r=1}^{3}\left(\frac{r}{7}\right) J_{1}^{(2 k)}(r \pi \tau \mid 7 \tau) .
$$

Proof. Logarithmically differentiating both sides of (10-1), we find that

$$
J_{1}\left(\frac{z}{\tau} \mid-\frac{1}{\tau}\right)=\frac{2 i z}{\pi}+\tau J_{1}(z \mid \tau) .
$$


Differentiate this equation $2 k(k \geq 1)$ times with respect to $z$ to deduce that

$$
J_{1}^{(2 k)}\left(\frac{z}{\tau} \mid-\frac{1}{\tau}\right)=\tau^{2 k+1} J_{1}^{(2 k)}(z \mid \tau) .
$$

We replace $\tau$ by $7 \tau$ in the above and then set $z=r \pi \tau$ to conclude that

$$
J_{1}^{(2 k)}\left(\frac{r \pi}{7} \mid-\frac{1}{7 \tau}\right)=(7 \tau)^{2 k+1} J_{1}^{(2 k)}(r \pi \tau \mid 7 \tau) .
$$

Multiply this equation by the Legendre symbol $\left(\frac{r}{7}\right)$ and then sum the resulting equation with respect to $r$ over $\{1,2,3\}$ to obtain the proposition.

Now we will apply Proposition 12.5 to derive the following identity of Ramanujan [Ramanujan 1988, page 145; Raghavan 1986].

Proposition 12.6. Let $\lambda, \mu$ be defined as in (1-3). Then we have

$$
\sum_{n=1}^{\infty}\left(\frac{n}{7}\right) \frac{q^{n}\left(1+q^{n}\right)}{\left(1-q^{n}\right)^{3}}=\lambda^{-1}(\mu+8)
$$

Starting from this identity, Ramanujan [1988, page 145] derived the important identity

$$
\sum_{n=0}^{\infty} p(7 n+5) q^{n}=7 \prod_{n=1}^{\infty} \frac{\left(1-q^{7 n}\right)^{3}}{\left(1-q^{n}\right)^{3}}+49 q \prod_{n=1}^{\infty} \frac{\left(1-q^{7 n}\right)^{7}}{\left(1-q^{n}\right)^{8}},
$$

where $p(n)$ denotes the number of unrestricted partitions of the positive integer $n$. The identity above implies the Ramanujan congruence $p(7 n+5) \equiv 0(\bmod 7)$.

Proof. We compare Ramanujan's identity in Proposition 9.5 with the second identity in (12-2) to find that

$$
\sum_{r=1}^{3}\left(\frac{r}{7}\right) J_{1}^{\prime \prime}\left(\frac{r \pi}{7} \mid \tau\right)=\frac{8}{\sqrt{7}} \lambda^{-1}\left(8 \mu^{2}+49 \mu\right) .
$$

Replacing $\tau$ by $-1 /(7 \tau)$ and then applying the modular transformation formulas for $\lambda$ and $\mu$ in (12-4), we deduce that

$$
\sum_{r=1}^{3}\left(\frac{r}{7}\right) J_{1}^{\prime \prime}\left(\frac{r \pi}{7} \mid-\frac{1}{7 \tau}\right)=2744 i \tau^{3} \lambda^{-1}(\mu+8) .
$$

Using the case $k=1$ of Proposition 12.5 in the left hand side of this equation, we find that $\sum_{r=1}^{3}\left(\frac{r}{7}\right) J_{1}^{\prime \prime}(r \pi \tau \mid 7 \tau)=8 i \tau^{3} \lambda^{-1}(\mu+8)$. If we substitute the fifth identity in (12-2) into this equation, we conclude that

$$
\sum_{r=1}^{6}\left(\frac{r}{7}\right) \sum_{n=1}^{\infty} \frac{n^{2} q^{r n}}{1-q^{7 n}}=\lambda^{-1}(\mu+8),
$$


which is equivalent to the Ramanujan identity in Proposition 12.6.

In [Liu 2003, (1.18)], we established the identity

$$
16+\sum_{n=1}^{\infty}\left(\frac{n}{7}\right) \frac{n^{4} q^{n}}{1-q^{n}}=\lambda^{-5 / 3}\left(16 \mu^{2}+49 \mu\right)\left(\mu^{2}+13 \mu+49\right)^{2 / 3} .
$$

Using the same argument used to get the Ramanujan identity in Proposition 12.6 from Proposition 9.5, we can deduce from (12-7) the following proposition.

Proposition 12.7. $\sum_{r=1}^{6}\left(\frac{r}{7}\right) \sum_{n=1}^{\infty} \frac{n^{4} q^{r n}}{1-q^{7 n}}=\lambda^{-5 / 3}(16+\mu)\left(\mu^{2}+13 \mu+49\right)^{2 / 3}$.

Now we are ready to prove the last two identities in Theorem 1.17 by employing Theorem 1.16.

Proof. Setting $x=\pi / 7$ and $y=2 \pi / 7$ in Theorem 1.16, we immediately have

$$
\begin{aligned}
16 E_{6}(\tau)+\sum_{r=1}^{3} J_{r}^{(5)}\left(\frac{r \pi}{7} \mid \tau\right)= & 40\left(\sum_{r=1}^{3}\left(\frac{r}{7}\right) J_{1}\left(\frac{r \pi}{7} \mid \tau\right)\right)^{3}\left(\sum_{r=1}^{3}\left(\frac{r}{7}\right) J_{1}^{\prime \prime}\left(\frac{r \pi}{7} \mid \tau\right)\right) \\
& -6\left(\sum_{r=1}^{3}\left(\frac{r}{7}\right) J_{1}\left(\frac{r \pi}{7} \mid \tau\right)\right)\left(\sum_{r=1}^{3}\left(\frac{r}{7}\right) J_{1}^{(4)}\left(\frac{r \pi}{7} \mid \tau\right)\right) \\
- & 10\left(\sum_{r=1}^{3}\left(\frac{r}{7}\right) J_{1}^{\prime \prime}\left(\frac{r \pi}{7} \mid \tau\right)\right)^{2}-16\left(\sum_{r=1}^{3}\left(\frac{r}{7}\right) J_{1}\left(\frac{r \pi}{7} \mid \tau\right)\right)^{6} .
\end{aligned}
$$

Substituting the third identity in (12-1), and the first three identities in (12-2) into this equation, and then dividing both sides by 8 , we find that

$$
\begin{aligned}
\frac{1}{63}\left(127 E_{6}(\tau)-7^{6} E_{6}(7 \tau)\right)= & 280\left(1+2 \sum_{n=1}^{\infty}\left(\frac{n}{7}\right) \frac{q^{n}}{1-q^{n}}\right)^{3}\left(8-7 \sum_{n=1}^{\infty}\left(\frac{n}{7}\right) \frac{n^{2} q^{n}}{1-q^{n}}\right) \\
& -168\left(1+2 \sum_{n=1}^{\infty}\left(\frac{n}{7}\right) \frac{q^{n}}{1-q^{n}}\right)\left(16+\sum_{n=1}^{\infty}\left(\frac{n}{7}\right) \frac{n^{4} q^{n}}{1-q^{n}}\right) \\
- & \frac{80}{7}\left(8-7 \sum_{n=1}^{\infty}\left(\frac{n}{7}\right) \frac{n^{2} q^{n}}{1-q^{n}}\right)^{2}-686\left(1+2 \sum_{n=1}^{\infty}\left(\frac{n}{7}\right) \frac{q^{n}}{1-q^{n}}\right)^{6} .
\end{aligned}
$$

Substituting the two identities in Theorem 1.11 and Proposition 9.5, (12-7) and the identity in Proposition 12.7 into the right side of the equation above, and simplifying, we conclude that

(12-8) $\quad \frac{1}{63}\left(127 E_{6}(\tau)-7^{6} E_{6}(7 \tau)\right)$

$$
=-\lambda^{-2}\left(\frac{13058}{7} \mu^{4}+27132 \mu^{3}+161210 \mu^{2}+605052 \mu+1647086\right) .
$$


The modular transformation formula for $E_{6}$ [Apostol 1990, page 24] is

$$
E_{6}(-1 / \tau)=\tau^{6} E_{6}(\tau)
$$

Replacing $\tau$ with $-1 /(7 \tau)$ in (12-8) and then using the modular transformation formulas for $E_{6}, \lambda$, and $\mu$ in (12-4) and (12-8), we deduce that

$$
\text { 0) } \frac{1}{63}\left(127 E_{6}(7 \tau)-E_{6}(\tau)\right)=\lambda^{-2}\left(2 \mu^{4}+36 \mu^{3}+470 \mu^{2}+3876 \mu+13058\right) \text {. }
$$

By solving the equations (12-8) and (12-10), we obtain the last two identities in Theorem 1.17, completing the proof.

\section{Some trigonometric series identities}

Proof of Theorems 1.18 and 1.19. We begin our proof by recalling the relation $\theta_{1}(z+\pi \tau / 2 \mid \tau)=i q^{-1 / 8} e^{-i z} \theta_{4}(z \mid \tau)$ satisfied by $\theta_{1}$ and $\theta_{4}$. Logarithmically differentiating this identity, we are led to

$$
J_{1}(z+\pi \tau / 2 \mid \tau)=-i+J_{4}(z \mid \tau), \quad J_{1}^{(k)}(z+\pi \tau / 2 \mid \tau)=J_{4}^{(k)}(z \mid \tau) \quad \text { for } k \geq 1 .
$$

Taking $y=\pi \tau / 2-x$ in Theorem 1.15, applying these two equations in the resulting equation, and noting that $J_{4}(0 \mid \tau)=J_{4}^{\prime \prime}(0 \mid \tau)=0$, we find that

$$
\begin{aligned}
& 2 E_{4}(\tau)+J_{1}^{\prime \prime \prime}(x \mid \tau)+J_{4}^{\prime \prime \prime}(x \mid \tau)+J_{4}^{\prime \prime \prime}(0 \mid \tau) \\
& =2\left(J_{1}(x \mid \tau)-J_{4}(x \mid \tau)\right)^{4}-4\left(J_{1}(x \mid \tau)-J_{4}(x \mid \tau)\right)\left(J_{1}^{\prime \prime}(x \mid \tau)-J_{4}^{\prime \prime}(x \mid \tau)\right) .
\end{aligned}
$$

Appealing to the trigonometric series expansions of $J_{1}$ and $J_{4}$ in (3-10) and (4-14), we find that

$$
\begin{aligned}
J_{1}(x \mid \tau)-J_{4}(x \mid \tau) & =\cot x-4 \sum_{n=1}^{\infty} \frac{q^{n / 2}}{1+q^{n / 2}} \sin 2 n x, \\
J_{1}^{\prime \prime}(x \mid \tau)-J_{4}^{\prime \prime}(x \mid \tau) & =2 \cot x+2 \cot ^{3} x+16 \sum_{n=1}^{\infty} \frac{n^{2} q^{n / 2}}{1+q^{n / 2}} \sin 2 n x, \\
J_{1}^{\prime \prime \prime}(x \mid \tau)+J_{4}^{\prime \prime \prime}(x \mid \tau) & =-2-8 \cot ^{2} x-6 \cot ^{4} x-32 \sum_{n=1}^{\infty} \frac{n^{3} q^{n / 2}}{1-q^{n / 2}} \cos 2 n x, \\
J_{4}^{\prime \prime \prime}(0 \mid \tau) & =-32 \sum_{n=1}^{\infty} \frac{n^{3} q^{n / 2}}{1-q^{n}} .
\end{aligned}
$$

Substituting these four equations into (13-1) and replacing $q$ by $q^{2}$ and $x$ by $z$, we are led to Theorem 1.18.

Similarly, by taking $y=\pi / 2-x$ in Theorem 1.15 and proceeding through the same steps as in deducing Theorem 1.18 from Theorem 1.15, we can obtain Theorem 1.19. 


\section{A theta function identity involving theta functions and the $\eta$-function}

Proof of Theorem 1.20. To prove Theorem 1.20 we construct the function

$$
F(z)=\frac{\theta_{1}(3 z \mid \tau) \theta_{1}^{2}(z \mid \tau)}{\theta_{1}(2 z \mid \tau) \theta_{1}(7 z \mid 7 \tau)}
$$

It is easy to verify that $F(z)$ is an elliptic function with periods $\pi$ and $\pi \tau$. It is easily seen that

$$
z_{1}=\frac{\pi}{2}, \quad z_{2}=\frac{\pi+\pi \tau}{2}, \quad z_{3}=\frac{\pi \tau}{2}, \quad z_{4}=\frac{\pi}{7}, \quad z_{5}=\frac{2 \pi}{7}, \ldots, z_{9}=\frac{6 \pi}{7}
$$

are the only poles of $F(z)$ and that all these poles are simple. By direct and elementary calculations, we find that

$$
\operatorname{res}\left(F ; z_{1}\right)=\lim _{z \rightarrow \pi / 2}(z-\pi / 2) \times \frac{\theta_{1}(3 z \mid \tau) \theta_{1}^{2}(z \mid \tau)}{\theta_{1}(2 z \mid \tau) \theta_{1}(7 z \mid 7 \tau)}=-\frac{\theta_{2}^{3}(0 \mid \tau)}{4 \eta^{3}(\tau) \theta_{2}(0 \mid 7 \tau)} .
$$

In the same way, we have

$$
\begin{aligned}
& \operatorname{res}\left(F ; z_{2}\right)=\frac{\theta_{3}^{3}(0 \mid \tau)}{4 \eta^{3}(\tau) \theta_{3}(0 \mid 7 \tau)}, \\
& \operatorname{res}\left(F ; z_{3}\right)=-\frac{\theta_{4}^{3}(0 \mid \tau)}{4 \eta^{3}(\tau) \theta_{4}(0 \mid 7 \tau)} \\
& \operatorname{res}\left(F ; z_{4}\right)=\operatorname{res}\left(F ; z_{9}\right)=-\frac{\eta^{2}(\tau)}{\left.2 \sqrt{7} \eta^{2}(7 \tau)\right)} \frac{\theta_{1}(\pi / 7 \mid \tau)}{\theta_{1}^{2}(2 \pi / 7 \mid \tau)} \\
& \operatorname{res}\left(F ; z_{5}\right)=\operatorname{res}\left(F ; z_{8}\right)=\frac{\eta^{2}(\tau)}{2 \sqrt{7} \eta^{2}(7 \tau)} \frac{\theta_{1}(2 \pi / 7 \mid \tau)}{\theta_{1}^{2}(3 \pi / 7 \mid \tau)} \\
& \operatorname{res}\left(F ; z_{6}\right)=\operatorname{res}\left(F ; z_{7}\right)=\frac{\eta^{2}(\tau)}{2 \sqrt{7} \eta^{2}(7 \tau)} \frac{\theta_{1}(3 \pi / 7 \mid \tau)}{\theta_{1}^{2}(\pi / 7 \mid \tau)}
\end{aligned}
$$

Substituting these equations into the identity $\sum_{k=1}^{9} \operatorname{res}\left(F ; z_{k}\right)=0$ and simplifying, we conclude that

$$
\begin{aligned}
\frac{\theta_{2}^{3}(0 \mid \tau)}{\theta_{2}(0 \mid 7 \tau)}-\frac{\theta_{3}^{3}(0 \mid \tau)}{\theta_{3}(0 \mid 7 \tau)} & +\frac{\theta_{4}^{3}(0 \mid \tau)}{\theta_{4}(0 \mid 7 \tau)} \\
& =\frac{4 \eta^{5}(\tau)}{\sqrt{7} \eta^{2}(7 \tau)}\left(\frac{\theta_{1}(3 \pi / 7 \mid \tau)}{\theta_{1}^{2}(\pi / 7 \mid \tau)}-\frac{\theta_{1}(\pi / 7 \mid \tau)}{\theta_{1}^{2}(2 \pi / 7 \mid \tau)}+\frac{\theta_{1}(2 \pi / 7 \mid \tau)}{\theta_{1}^{2}(3 \pi / 7 \mid \tau)}\right) .
\end{aligned}
$$

In view the definitions of $u, v$, and $w$ in (8-1), we find (8-19) can be rewritten as $\left(\frac{\theta_{1}(3 \pi / 7 \mid \tau)}{\theta_{1}^{2}(\pi / 7 \mid \tau)}-\frac{\theta_{1}(\pi / 7 \mid \tau)}{\theta_{1}^{2}(2 \pi / 7 \mid \tau)}+\frac{\theta_{1}(2 \pi / 7 \mid \tau)}{\theta_{1}^{2}(3 \pi / 7 \mid \tau)}\right)^{3}=7 \sqrt{7} \eta^{-9}(\tau) \lambda^{-1}\left(\mu^{2}+13 \mu+49\right)$.

Combining the above two equations, we complete the proof of Theorem 1.20. 


\section{Acknowledgements}

I would like to thank the anonymous referee for many invaluable suggestions.

\section{References}

[Andrews et al. 1999] G. E. Andrews, R. Askey, and R. Roy, Special functions, Encyclopedia of Mathematics and its Applications 71, Cambridge University Press, 1999. MR 2000g:33001 Zbl 0920.33001

[Andrews et al. 2001] G. E. Andrews, R. Lewis, and Z.-G. Liu, "An identity relating a theta function to a sum of Lambert series", Bull. London Math. Soc. 33:1 (2001), 25-31. MR 2001k:11073 Zbl 1021.11010

[Apostol 1990] T. M. Apostol, Modular functions and Dirichlet series in number theory, 2nd ed., Graduate Texts in Mathematics 41, Springer, New York, 1990. MR 90j:11001 Zbl 0697.10023

[Beck et al. 2005] M. Beck, B. C. Berndt, O.-Y. Chan, and A. Zaharescu, "Determinations of analogues of Gauss sums and other trigonometric sums", Int. J. Number Theory 1:3 (2005), 333-356. MR 2006e:11114 Zbl 1088.11064

[Berndt 1991] B. C. Berndt, Ramanujan's notebooks, Part III, Springer, New York, 1991. MR 92j: 01069 Zbl 0733.11001

[Berndt 2006] B. C. Berndt, Number theory in the spirit of Ramanujan, Student Mathematical Library 34, American Mathematical Society, Providence, RI, 2006. MR 2007f:11001 Zbl 1117.11001

[Berndt and Yee 2003] B. C. Berndt and A. J. Yee, "A page on Eisenstein series in Ramanujan's Lost Notebook", Glasg. Math. J. 45:1 (2003), 123-129. MR 2004a:11032 Zbl 1090.11025

[Berndt and Zaharescu 2004] B. C. Berndt and A. Zaharescu, "Finite trigonometric sums and class numbers”, Math. Ann. 330:3 (2004), 551-575. MR 2005f:11173 Zbl 1099.11039

[Berndt and Zhang 1994] B. C. Berndt and L. C. Zhang, "A new class of theta-function identities originating in Ramanujan's notebooks”, J. Number Theory 48:2 (1994), 224-242. MR 95m:11046 Zbl 0812.11028

[Berndt et al. 2000] B. C. Berndt, H. H. Chan, J. Sohn, and S. H. Son, "Eisenstein series in Ramanujan's lost notebook", Ramanujan J. 4:1 (2000), 81-114. MR 2001j:11018 Zbl 1024.11026

[Chan 2007] O.-Y. Chan, "Weighted trigonometric sums over a half-period", Adv. in Appl. Math. 38:4 (2007), 482-504. MR 2008a:11092 Zbl 1151.11040

[Chan and Cooper 2008] H. H. Chan and S. Cooper, "Eisenstein series and theta functions to the septic base", J. Number Theory 128:3 (2008), 680-699. MR 2009c:33050 Zbl 05242980

[Cooper 2006] S. Cooper, "The quintuple product identity", Int. J. Number Theory 2:1 (2006), 115161. MR 2007f:33021 Zbl 1159.33300

[Elkies 1999] N. D. Elkies, "The Klein quartic in number theory", pp. 51-101 in The eightfold way, edited by S. Levy, Math. Sci. Res. Inst. Publ. 35, Cambridge Univ. Press, 1999. MR 2001a:11103 Zbl 0991.11032

[Enneper 1890] V. Enneper, Elliptische Functionen: Theorie und Geschichte, Louis Nebert, Halle a.S., 1890. JFM 21.0455.01

[Fine 1956] N. Fine, "On a system of modular functions connected with the Ramanujan identities", Tôhoku Math. J. (2) 8 (1956), 149-164. MR 19,392c Zbl 0073.02904

[Garvan 1984] F. G. Garvan, “A simple proof of Watson's partition congruences for powers of 7”, $J$. Austral. Math. Soc. Ser. A 36:3 (1984), 316-334. MR 85f:11072 Zbl 0533.10006

[Guetzlaff 1834] C. Guetzlaff, "Aequatio modularis pro transformatione functionum ellipticarum septimi ordinis”, J. Reine Angew. Math. 12 (1834), 173-177. 
[Hardy and Wright 1979] G. H. Hardy and E. M. Wright, An introduction to the theory of numbers, 5th ed., Oxford University Press, New York, 1979. MR 81i:10002 Zbl 0423.10001

[Hirschhorn 1987] M. D. Hirschhorn, "A simple proof of Jacobi's four-square theorem", Proc. Amer. Math. Soc. 101:3 (1987), 436-438. MR 88m:11084 Zbl 0632.10046

[Klein 1999] F. Klein, "On the order-seven transformation of elliptic functions”, pp. 287-331 in The eightfold way, edited by S. Levy, Math. Sci. Res. Inst. Publ. 35, Cambridge Univ. Press, 1999. MR 2001i:14042 Zbl 0972.14022

[Lachaud 2005] G. Lachaud, "Ramanujan modular forms and the Klein quartic", Mosc. Math. J. 5:4 (2005), 829-856, 972-973. MR 2008e:11074 Zbl 1131.11040

[Liu 2001] Z.-G. Liu, "Residue theorem and theta function identities", Ramanujan J. 5:2 (2001), 129-151. MR 2002g:11056 Zbl 1011.11025

[Liu 2003] Z.-G. Liu, "Some Eisenstein series identities related to modular equations of the seventh order”, Pacific J. Math. 209:1 (2003), 103-130. MR 2004c:11052 Zbl 1050.11048

[Liu 2005] Z.-G. Liu, "A three-term theta function identity and its applications", Adv. Math. 195:1 (2005), 1-23. MR 2006c:11050 Zbl 1138.11014

[Liu 2007] Z.-G. Liu, "An addition formula for the Jacobian theta function and its applications", Adv. Math. 212:1 (2007), 389-406. MR 2008c:11065 Zbl 1162.11025

[Liu 2009] Z.-G. Liu, “Addition formulas for Jacobi theta functions, Dedekind's eta function, and Ramanujan's congruences”, Pacific J. Math. 240:1 (2009), 135-150. MR 2010a:33057 Zbl 1169. 33005

[McCullough and Shen 1994] S. McCullough and L.-C. Shen, "On the Szegó kernel of an annulus", Proc. Amer. Math. Soc. 121:4 (1994), 1111-1121. MR 94j:30005

[Milne 2002] S. C. Milne, "Infinite families of exact sums of squares formulas, Jacobi elliptic functions, continued fractions, and Schur functions", Ramanujan J. 6:1 (2002), 7-149. MR 2003m: $11060 \mathrm{Zbl} 1125.11315$

[Raghavan 1986] S. Raghavan, "On certain identities due to Ramanujan", Quart. J. Math. Oxford Ser. (2) 37:146 (1986), 221-229. MR 87j:11113 Zbl 0585.10033

[Raghavan and Rangachari 1989] S. Raghavan and S. S. Rangachari, "On Ramanujan's elliptic integrals and modular identities", pp. 119-149 in Number theory and related topics (Bombay, 1988), Fund. Res. Stud. Math. 12, Tata Inst., Bombay, 1989. MR 98b:11045 Zbl 0748.33013

[Ramanujan 1988] S. Ramanujan, The lost notebook and other unpublished papers, Springer, Berlin, 1988. MR 89j:01078 Zbl 0639.01023

[Watson 1938] G. Watson, "Ramanujans Vermutung über Zerfällungsanzahlen”, J. Reine Angew. Math. 179 (1938), 97-128. Zbl 0019.15302

[Whittaker and Watson 1996] E. T. Whittaker and G. N. Watson, A course of modern analysis, Cambridge University Press, 1996. MR 97k:01072 Zbl 0951.30002

Received April 2, 2009.

\section{ZHI-GUO LIU}

DEPARTMENT OF MATHEMATICS

EAST CHINA NORMAL UNIVERSITY

500 DONGCHUAN ROAD

SHANGHAI, 200241

CHINA

zgliu@math.ecnu.edu.cn 


\title{
PACIFIC JOURNAL OF MATHEMATICS
}

\author{
http://www.pjmath.org \\ Founded in 1951 by \\ E. F. Beckenbach (1906-1982) and F. Wolf (1904-1989)
}

\section{EDITORS}

V. S. Varadarajan (Managing Editor)

Department of Mathematics

University of California

Los Angeles, CA 90095-1555

pacific@math.ucla.edu

Vyjayanthi Chari

Department of Mathematics

University of California

Riverside, CA 92521-0135

chari@math.ucr.edu

Robert Finn

Department of Mathematics Stanford University

Stanford, CA 94305-2125

finn@math.stanford.edu

Kefeng Liu

Department of Mathematics

University of California

Los Angeles, CA 90095-1555

liu@math.ucla.edu
Paulo Ney de Souza, Production Manager

ACADEMIA SINICA, TAIPEI

CALIFORNIA INST. OF TECHNOLOGY

INST. DE MATEMÁTICA PURA E APLICADA

KEIO UNIVERSITY

MATH. SCIENCES RESEARCH INSTITUTE

NEW MEXICO STATE UNIV.

OREGON STATE UNIV.

\section{Darren Long}

Department of Mathematics

University of California

Santa Barbara, CA 93106-3080

long@math.ucsb.edu

Jiang-Hua Lu

Department of Mathematics

The University of Hong Kong

Pokfulam Rd., Hong Kong

jhlu@maths.hku.hk

Alexander Merkurjev

Department of Mathematics

University of California

Los Angeles, CA 90095-1555

merkurev@math.ucla.edu

\section{PRODUCTION}

pacific@math.berkeley.edu

Silvio Levy, Senior Production Editor

\section{SUPPORTING INSTITUTIONS}

STANFORD UNIVERSITY
UNIV. OF BRITISH COLUMBIA
UNIV. OF CALIFORNIA, BERKELEY
UNIV. OF CALIFORNIA, DAVIS
UNIV. OF CALIFORNIA, LOS ANGELES
UNIV. OF CALIFORNIA, RIVERSIDE
UNIV. OF CALIFORNIA, SAN DIEGO
UNIV. OF CALIF., SANTA BARBARA

Sorin Popa

Department of Mathematics University of California

Los Angeles, CA 90095-1555 popa@math.ucla.edu

Jie Qing

Department of Mathematics

University of California

Santa Cruz, CA 95064

qing@cats.ucsc.edu

Jonathan Rogawski

Department of Mathematics

University of California

Los Angeles, CA 90095-1555

jonr@math.ucla.edu

Matthew Cargo, Production Editor

UNIV. OF CALIF., SANTA CRUZ

UNIV. OF MONTANA

UNIV. OF OREGON

UNIV. OF SOUTHERN CALIFORNIA

UNIV. OF UTAH

UNIV. OF WASHINGTON

WASHINGTON STATE UNIVERSITY

These supporting institutions contribute to the cost of publication of this Journal, but they are not owners or publishers and have no responsibility for its contents or policies.

See inside back cover or www.pjmath.org for submission instructions.

The subscription price for 2010 is US \$420/year for the electronic version, and \$485/year for print and electronic.

Subscriptions, requests for back issues from the last three years and changes of subscribers address should be sent to Pacific Journal of Mathematics, P.O. Box 4163, Berkeley, CA 94704-0163, U.S.A. Prior back issues are obtainable from Periodicals Service Company, 11 Main Street, Germantown, NY 12526-5635. The Pacific Journal of Mathematics is indexed by Mathematical Reviews, Zentralblatt MATH, PASCAL CNRS Index, Referativnyi Zhurnal, Current Mathematical Publications and the Science Citation Index.

The Pacific Journal of Mathematics (ISSN 0030-8730) at the University of California, c/o Department of Mathematics, 969 Evans Hall, Berkeley, CA 94720-3840, is published monthly except July and August. Periodical rate postage paid at Berkeley, CA 94704, and additional mailing offices. POSTMASTER: send address changes to Pacific Journal of Mathematics, P.O. Box 4163, Berkeley, CA 94704-0163.

PJM peer-review and production is managed by EditFLOW ${ }^{\mathrm{TM}}$ from Mathematical Sciences Publishers.

PUBLISHED BY PACIFIC JOURNAL OF MATHEMATICS

at the University of California, Berkeley $94720-3840$

A NON-PROFIT CORPORATION

Typeset in LATEX

Copyright $@ 2010$ by Pacific Journal of Mathematics 


\section{PACIFIC JOURNAL OF MATHEMATICS}

Volume $246 \quad$ No. $2 \quad$ June 2010

Geometric structures associated to a contact metric $(\kappa, \mu)$-space

Beniamino CAPPELLETTI MONTANO and LUigia Di TERLizzi

Multilinear singular operators with fractional rank

Ciprian Demeter, Malabika PramaniK and Christoph Thiele

A new proof of Reifenberg's topological disc theorem

GUANGHAO HONG and LiHE WANG

Global classical solutions to hyperbolic geometric flow on Riemann surfaces

FAGUi LiU and YUANZHANG ZHANG

An extension of the quintuple product identity and its applications

ZHI-GUO LIU

A generalization of the Pontryagin-Hill theorems to projective modules over

Prüfer domains

JORGE MACÍAS-DíAZ

Elliptic pseudodifferential equations and Sobolev spaces over $p$-adic fields

J. J. RodRíGUEZ-VEGA and W. A. ZÚÑIGA-GALINDO

Absolutely isolated singularities of holomorphic maps of $\mathbb{C}^{n}$ tangent to the identity

\section{FENG RONG}

Pullbacks of Eisenstein series from $\mathrm{GU}(3,3)$ and critical $L$-values for

$\mathrm{GSp}(4) \times \mathrm{GL}(2)$

\section{ABHISHEK SAHA}

Isomorphism invariants of restricted enveloping algebras

HAMID USEFI

Spacelike S-Willmore spheres in Lorentzian space forms

PENG WANG 